\title{
ngularly Perturbed and Nonlocal Modulation Equations for Systems with eracting Instability Mechanisms
}

elman, A.; Rottschäfer, V.

\section{blication date}

97

blished in

urnal of Nonlinear Science

$\mathrm{k}$ to publication

ation for published version (APA):

elman, A., \& Rottschäfer, V. (1997). Singularly Perturbed and Nonlocal Modulation uations for Systems with Interacting Instability Mechanisms. Journal of Nonlinear Scienc ), 371-409.

\section{eeral rights}

not permitted to download or to forward/distribute the text or part of it without the consent of the author(s) /or copyright holder(s), other than for strictly personal, individual use, unless the work is under an open tent license (like Creative Commons). he Library know, stating your reasons. In case of a legitimate complaint, the Library will make the material ccessible and/or remove it from the website. Please Ask the Library: https://uba.uva.nl/en/contact, or a lette Library of the University of Amsterdam, Secretariat, Singel 425, 1012 WP Amsterdam, The Netherlands. Y be contacted as soon as possible. 
(C) 1997 Springer-Verlag New York Inc.

\title{
Singularly Perturbed and Nonlocal Modulation Equations for Systems with Interacting Instability Mechanisms
}

\author{
A. Doelman and V. Rottschäfer \\ Mathematisch Instituut, Universiteit Utrecht, Postbus 80.010, 3508TA Utrecht, \\ The Netherlands \\ Received March 18, 1996; revised manuscript accepted for publication November 22, 1996 \\ Communicated by Phillip Holmes
}

Summary. Two related systems of coupled modulation equations are studied and compared in this paper. The modulation equations are derived for a certain class of basic systems which are subject to two distinct, interacting, destabilising mechanisms. We assume that, near criticality, the ratio of the widths of the unstable wavenumber-intervals of the two (weakly) unstable modes is small—as, for instance, can be the case in doublelayer convection. Based on these assumptions we first derive a singularly perturbed modulation equation and then a modulation equation with a nonlocal term. The reduction of the singularly perturbed system to the nonlocal system can be interpreted as a limit in which the width of the smallest unstable interval vanishes. We study and compare the behaviour of the stationary solutions of both systems. It is found that spatially periodic stationary solutions of the nonlocal system exist under the same conditions as spatially periodic stationary solutions of the singularly perturbed system. Moreover, these solutions can be interpreted as representing the same quasi-periodic patterns in the underlying basic system. Thus, the 'Landau reduction' to the nonlocal system has no significant influence on the stationary quasi-periodic patterns. However, a large variety of intricate heteroclinic and homoclinic connections is found for the singularly perturbed system. These orbits all correspond to so-called 'localised structures' in the underlying system: They connect simple periodic patterns at $x \rightarrow \pm \infty$. None of these patterns can be described by the nonlocal system. So, one may conclude that the reduction to the nonlocal system destroys a rich and important set of patterns.

\section{Introduction}

In the weakly nonlinear stability theory of the evolution of patterns one classically considers systems like

$$
\psi_{t}=L_{R} \psi+N(\psi), \quad \psi(x, y, t): \mathbb{R}^{n} \times \Omega \times \mathbb{R}^{+} \rightarrow \mathbb{R}^{N},
$$




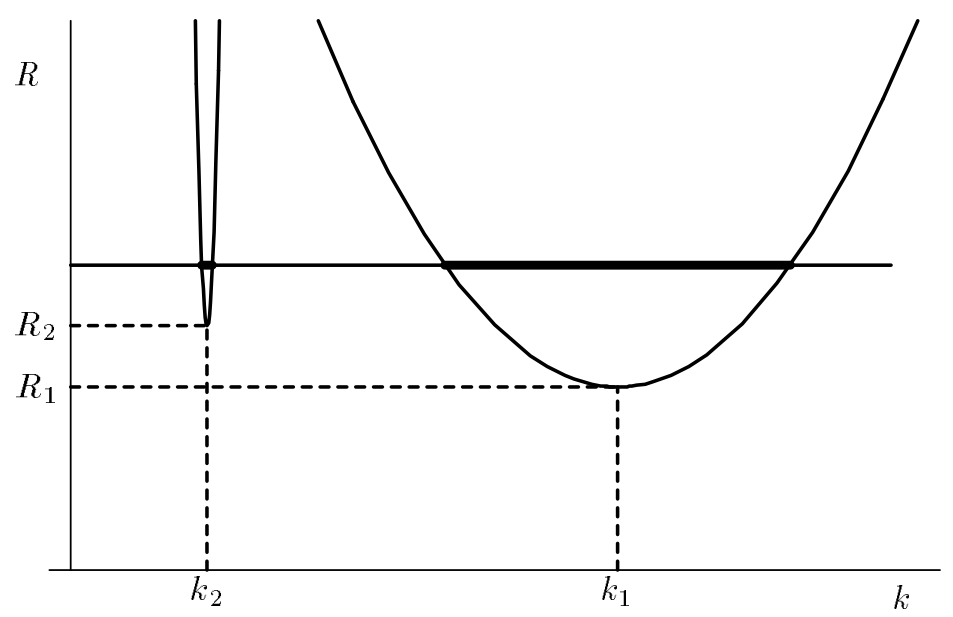

Fig. 1. A critical curve with two local minima, one at $\left(k_{1}, R_{1}\right)$ and the other at $\left(k_{2}, R_{2}\right)$. Here, $R$ is fixed at a value above $R_{1}$ and $R_{2}$ : There are two intervals of 'unstable waves.' The small parameter $\delta$ corresponds to the ratio of the widths of these intervals.

where $L_{R}$ (respectively $N$ ) is a linear (nonlinear) operator, $R$ is a control, or bifurcation, parameter, and $\Omega$ is a bounded domain $\subset \mathbb{R}^{m}$. We refer to the review paper by Eckhaus [9] for a survey and some of the numerous physical examples. This system is assumed to have a basic solution $\psi_{0}(y)$. The linearised stability of this solution is determined by setting

$$
\psi=\psi_{0}+f(y) e^{i k x+\mu t}
$$

and solving, for any pair $(k, R)$, an eigenvalue problem for $f(y)$ with eigenvalues $\mu=$ $\mu(k, R)$. Note that $k$ is a scalar: We have assumed that $n=1$ in (1.1) (for simplicity, we will assume $n=1$ throughout this paper). The neutral curve is defined as the set $\left\{\operatorname{Re} \mu_{0}(k, R)=0\right\}$, where $\mu_{0}(k, R)$ is the critical eigenvalue (that is, the eigenvalue with the largest real part) for a given pair $(k, R)$. The basic solution $\psi_{0}$ is linearly stable for $R=R_{0}$ if $\operatorname{Re} \mu_{0}\left(k, R_{0}\right)<0$ for all $k$. A bifurcation occurs if one increases $R$ such that $\{R=$ const. $\}$ intersects the neutral curve at a minimum $\left(k_{c}, R_{c}\right)$ of this curve: A small interval of 'linearly unstable waves' appears for $R>R_{c}$ (see Figure 1, where either $k_{1}$ or $k_{2}$ plays the role of $k_{c}$ ). To understand the behaviour of the solutions to (1.1) for $R$ close to $R_{c}$, or $R-R_{c}=r \varepsilon^{2}, 0<\varepsilon \ll 1$, one first shows that the nonlinear evolution of solutions close $(=O(\varepsilon))$ to $\psi_{0}$ is governed by the (complex) amplitude $A(\xi, \tau)$ of the linearly 'most unstable mode' $f_{c}(y) e^{i\left(k_{c} x+\mu_{c} t\right)}$, where $f_{c}(y)$ is the critical eigenfunction at the eigenvalue $\mu_{0}\left(k_{c}, R_{c}\right)=i \mu_{c} ; \xi$ and $\tau$ are slow spatial and temporal variables. Then, one derives an equation for $A(\xi, \tau)$, the so-called Ginzburg-Landau equation,

$$
A_{\tau}=r \mu_{R} A-\frac{1}{2} \mu_{k k} A_{\xi \xi}+c A|A|^{2}
$$

where $\mu_{R}=\frac{\partial \mu}{\partial R}\left(k_{c}, R_{c}\right), \mu_{k k}=\frac{\partial^{2} \mu}{\partial k^{2}}\left(k_{c}, R_{c}\right)$, and $c \in \mathbf{C}$ is the so-called Landau constant. Note that $\operatorname{Re} \mu_{R}>0$ and $\operatorname{Re} \mu_{k k}<0$ since the neutral curve can be approximated near 
the minimum $\left(k_{c}, R_{c}\right)$ by the parabola

$$
R=R_{c}-\frac{1}{2} \frac{\operatorname{Re} \mu_{k k}}{\operatorname{Re} \mu_{R}}\left(k-k_{c}\right)^{2} .
$$

Details of this procedure are, for instance, given in [9]. Recently, a number of papers on the mathematical validity of the Ginzburg-Landau approximation have appeared; we refer to [2] for a survey and relevant references. The Landau equation associated with this nonlinear stability problem can be obtained from (1.2) by setting $A(\xi, \tau)=A(\tau)$. This 'Landau reduction' can be interpreted by saying that one neglects the width of the band of unstable waves centred around $k=k_{c}$ for $R=R_{c}+r \varepsilon^{2}$. Historically, the Landau equation was derived a decade earlier than the Ginzburg-Landau equation (see [30]).

In this paper we consider a class of physical problems which have two distinct, interacting instability mechanisms at near-critical conditions. This means, in the above setting, that the neutral curve $\left\{\operatorname{Re} \mu_{0}(k, R)=0\right\}$ has two local minima, $\left(k_{1}, R_{1}\right)$ and $\left(k_{2}, R_{2}\right)$, such that $\left|R_{1}-R_{2}\right|$ is small (see Figure 1). So, if one chooses $R$ close to criticality in this case, one expects two independent, interacting, 'linearly most unstable waves,' $f_{1}(y) e^{i\left(k_{1} x+\mu_{1} t\right)}$ near $\left(k_{1}, R_{1}\right)$ with complex amplitude $A(\xi, \tau)$ and $f_{2}(y) e^{i\left(k_{2} x+\mu_{2} t\right)}$ near $\left(k_{2}, R_{2}\right)$ with amplitude $B(\xi, \tau)$. The nonlinear behaviour of patterns near criticality is then described by a coupled system of Ginzburg-Landau equations.

When a neutral curve has more than one local minimum one does not expect that those minima occur for (approximately) the same value of the bifurcation parameter $R$. However, the relative position of the minima can very often be changed as a second parameter $S$ is varied. Thus, by changing this second parameter $S$ the neutral curve transforms from a curve with an absolute minimum in $\left(k_{1}, R_{1}\right)$ to a curve with an absolute minimum $\left(k_{2}, R_{2}\right)$ (or vice versa). If $\left(k_{1}, R_{1}\right)$ is the absolute minimum then we are in the above described classical case and the evolution of patterns near criticality is governed by (1.2); if $\left(k_{2}, R_{2}\right)$ is the absolute minimum then the situation is again classical and is governed by a Ginzburg-Landau equation for $B(\xi, \tau)$. The two unstable modes interact in the transition region. This situation occurs in many applications and the coupled system of equations described above has been derived by many authors. We mention here some physical examples where two unstable modes can interact: double-layer convection [25], [24], [18]; crystal-growing experiments (where the convective and morphological modes can interact) [13], [26], [21]; gasless combustion [19]; sand ripple formation [31]. The coupled system of modulation equations has, for instance, been derived in [19], [17], [22].

In Section 2 we will give a short sketch of the derivation of the coupled system in the case of (nonresonantly) interacting instability mechanisms. The model problem considered in this paper is assumed to have a reflection symmetry in the one-dimensional unbounded variable $x$. Therefore, all coefficients in the coupled system of modulation equations will be real:

$$
\left\{\begin{array}{l}
A_{\tau}=r A+A_{\xi \xi}+A\left(t_{1}|A|^{2}+c_{1}|B|^{2}\right) \\
B_{\tau}=s B+D B_{\xi \xi}+B\left(t_{2}|B|^{2}+c_{2}|A|^{2}\right)
\end{array}\right.
$$

where $r$ and $s$ measure the distance between $R$ and $R_{1,2}$ (see Section 2 for more details). By rescaling, we have simplified the coefficients of the linear terms. Due to the reflection symmetry - which for instance occurs naturally in convection experiments - the 
$\xi$-variable is not moving (it is independent of $t$ ). This is a consequence of the fact that all eigenvalues $\mu(k, R)$ are real. If this is not the case, then the $A$ and $B$ amplitudes will be travelling with the group speed of the linearly unstable waves. This speed is in general not the same for the $A$ - and $B$-modes, so the interaction of the $A$ and $B$ patterns cannot be described by (1.4). In this case one has to apply some kind of averaging formalism in order to derive a so-called mean field Ginzburg-Landau equation; see for instance [17], [19], and [23] for a validity result.

Here, we focus on the significance of the diffusion parameter $D(>0)$ in (1.4): $D$ measures the relative widths of the bands of unstable modes just above the minima $\left(k_{1}, R_{1}\right)$ and $\left(k_{2}, R_{2}\right)$. More precisely, as in (1.2), the diffusion coefficients are determined by $\frac{\partial^{2} \mu}{\partial k^{2}}\left(k_{1,2}, R_{1,2}\right)$, which measure the curvature of the neutral curve at the minimum (see (1.3)). We have rescaled the diffusion coefficient in the $A$-equation to 1: $D$ measures the ratio of $\frac{\partial^{2} \mu}{\partial k^{2}}\left(k_{2}, R_{2}\right)$ and $\frac{\partial^{2} \mu}{\partial k^{2}}\left(k_{1}, R_{1}\right)$. Thus, $D \gg 1$ means that the neutral curve near $\left(k_{2}, R_{2}\right)$ is much 'sharper,' or narrower, than near $\left(k_{1}, R_{1}\right)$ (see Figure 1 and Section 2 for more details). This occurs for instance naturally in experiments on double-layer convection (where the depth of the layers differs significantly) and in experiments on crystal-growth (see [13], [25], and [22], [18] for a discussion). If this is the case one can introduce, apart from $\varepsilon \sim \sqrt{\left|R_{1}-R_{2}\right|}$, a second small parameter $0<\delta \ll 1$ by setting $D=\frac{1}{\delta^{2}}$ and write down a singularly perturbed system,

$$
\left\{\begin{array}{l}
A_{\tau}=r A+A_{\xi \xi}+A\left(t_{1}|A|^{2}+c_{1}|B|^{2}\right) \\
B_{\tau}=s B+\frac{1}{\delta^{2}} B_{\xi \xi}+B\left(t_{2}|B|^{2}+c_{2}|A|^{2}\right)
\end{array}\right.
$$

There is another, equivalent, way of interpreting this singular term: Both instability mechanisms are associated with a natural spatial scale at which the patterns evolve. In this paper we consider the case in which the magnitudes of these scales differ significantly. Due to the rescaling we can say that the natural scale associated with $A$ is $\xi$, while it is $\delta \xi$ for $B: B(\xi, \tau)$ only varies very slowly on the $\xi$-scale. Returning to the above interpretation this means that the width of the $\left(k_{2}, R_{2}\right)$-parabola (see $\left.(1.3)\right)$ is $O(\delta)$ compared to the width of the $\left(k_{1}, R_{1}\right)$-parabola (Figure 1). In this situation it is natural to apply the above-described Landau reduction for $B: B(\xi, \tau)=B(\tau)$. In Section 2 we show that (1.5) then reduces to the following nonlocal system:

$$
\left\{\begin{array}{l}
A_{\tau}=r A+A_{\xi \xi}+A\left(t_{1}|A|^{2}+c_{1}|B|^{2}\right), \\
B_{\tau}=s B+B\left(t_{2}|B|^{2}+c_{2} \lim _{M \rightarrow \infty} \frac{1}{2 M} \int_{-M}^{M}|A|^{2} d \xi\right) .
\end{array}\right.
$$

We shall also show that this reduction is only valid when $A(\xi, \tau)$ (and $B(\tau)$ ) satisfy an extra solvability condition,

$$
c_{2} B \int_{-\infty}^{\infty}\left(|A|^{2}-\lim _{M \rightarrow \infty} \frac{1}{2 M} \int_{-M}^{M}|A|^{2} d \hat{\xi}\right) e^{i K \xi} d \xi=O\left(\varepsilon^{2}\right) \quad \text { for } K=O(\delta) .
$$

This condition cannot be satisfied by all solutions of (1.6) (see Section 3.2). The idea of a Landau reduction has also been applied by Metzener and Proctor [22] in their analysis of the evolution of patterns at 'disparate scales.' Note that our approach is not exactly the same as in [22]: There $k_{2}$, instead of $D$, has been taken as a small parameter. In Section 2 we relate our approach to the one in [22]. Some fundamental properties of a modulation 
equation with a nonlocal term, such as the existence, uniqueness, regularity of solutions, and the dimension of attractors, have been studied in [7].

The main goal of this paper is to understand the impact of this Landau reduction for $B$ on the patterns described by (1.5). We focus on the analysis of the stationary solutions of (1.5) and (1.6). First we search for spatially periodic solutions. For both systems, the analysis is based on the fact that the (stationary) equation for $A$ is integrable when $B$ is fixed at a constant value (see [4] for references to the stationary problem of the (uncoupled) 'real' Ginzburg-Landau equation). Thus, the stationary problem associated with (1.5) is a (singularly) perturbed integrable system; periodic orbits in the fast field can be found by constructing a Poincare map. We find that both systems have a similar set of periodic solutions which exists under the same conditions on the parameters and, most importantly, which describe - up to $\mathcal{O}(\delta)$ corrections - the same family of quasi-periodic patterns in the basic system. The most important difference between the quasi-periodic patterns described by (1.5) and (1.6) is that $|B|$ is periodic with an $O(\delta)$ amplitude around a certain value $b$ in (1.5), while the corresponding solution described by (1.6) has $|B| \equiv b$. Thus, the above Landau reduction for the $B$-mode has no significant influence here.

By introducing polar coordinates for $A$ and $B$ it is possible to write the stationary singularly perturbed problem as a four-dimensional ODE with two fast directions, $x$ and $y$ corresponding to $A$, and two slow directions, $z$ and $w$ corresponding to $B$ :

$$
\left\{\begin{array}{l}
\dot{x}=y, \\
\dot{y}=-x+x\left(x^{2}-c_{1} z^{2}\right) \\
\dot{z}=\delta w \\
\dot{w}=\delta\left(-s z+z\left(z^{2}-c_{2} x^{2}\right)\right)
\end{array}\right.
$$

where we have scaled $\left(r, t_{1}, t_{2}\right)$ in $(1.5)$ to $(1,-1,-1)$ (thus we chose $t_{1,2}<0$, as occurs most frequently in applications); the 'dot' refers to differentiation with respect to 'time' $t$, where $t$ is now a rescaled version of $\xi$. The reduction from the expected eight-dimensional system to a four-dimensional system is due to the phase invariance in the equations for $A$ and $B$ and to the fact that there are two integrals, $\Omega_{1}$ and $\Omega_{2}$, in the full system. These integrals are uncoupled, in the sense that they are identical to the integrals of the uncoupled equations for $A$ and $B$ (see Section 4 and [4]). In deriving (1.8) we chose $\Omega_{1}=\Omega_{2}=0$ : This only simplifies the analysis of the four-dimensional system. Apart from other solutions, both 'most stable' (see [20]) Stokes-wave solutions, ( $A=$ const., $B \equiv 0$ ) and ( $B=$ const., $A \equiv 0$ ), satisfy $\Omega_{1}=\Omega_{2}=0$ and are thus described by (1.8). This four-dimensional system can be analysed (for instance) by the geometric theory for singularly perturbed systems, originally developed by Fenichel [11]; see also the contribution of Jones to [1]. Using the results of Fenichel we establish the existence of two so-called slow, invariant manifolds $\Gamma_{l}$ and $\Gamma_{r}$. We find a very rich structure of heteroclinic and homoclinic orbits which 'jump up and down' between $\Gamma_{l}$ and $\Gamma_{r}$. More precisely, there are four critical points on the slow manifolds: $P_{l}, Q_{l} \in \Gamma_{l}$ and $P_{r}, Q_{r} \in \Gamma_{r}$. For any $N>0$ there are $\mathcal{N}(N)$ different ' $N$-jump' heteroclinic or homoclinic orbits which connect two of the above four points and which consist of $N+1$ slow parts near $\Gamma_{l}$ or $\Gamma_{r}$ and $N$ jumps through the fast field. The number $\mathcal{N}(N)$ can be explicitly calculated: $\mathcal{N}(N)=4 \times$ the $(N+2)$-th Fibonacci number (see Theorems 1 and 2 in Sections 5.2 and 5.3). These results are obtained by carefully tracking the three- 
dimensional stable and unstable manifolds of $\Gamma_{l}, \Gamma_{r}$ and the two-dimensional stable and unstable manifolds of $P_{l, r}, Q_{l, r}$ through the fast field and near the slow manifolds. The Hamiltonian structure of (1.8) (see Section 4) is a key ingredient of the proof of our results. Based on the methods developed in [6] we are also able to show the existence of homoclinic orbits which do not jump immediately from $\Gamma_{l}$ to $\Gamma_{r}$ (or vice versa), but remain in the fast field for a 'longer time' (see Theorem 3 ).

These orbits all correspond to so-called 'localised structures' in the underlying system: they connect simple, spatially periodic patterns at $x \rightarrow \pm \infty$. Such localised structures can be stable in the uncoupled Ginzburg-Landau equation (see for instance [28] for a survey). However, none of these patterns can be described by the nonlocal reduction (1.6). There are two reasons for this. The first reason is that the most important ingredient of the construction of the heteroclinic and homoclinic orbits is the existence of the slow manifolds $\Gamma_{l}$ and $\Gamma_{r}$. These manifolds can (of course) not exist in the Landau reduction since $B$, and thus $z$ and $w$ in (1.8), cannot evolve slowly. However, there exist a small number of heteroclinic orbits in the stationary problem associated with (1.6) which do have a counterpart in (1.8): The orbits only remain near $\Gamma_{l, r}$ for an $O(\delta)$ distance. These 'localised patterns' cannot be described by the Landau reduction due to a second, independent reason: They do not satisfy the extra solvability condition (1.7); see Section 3.2.

Thus we conclude that the reduction to a Landau approach for $B$ destroys a rich and important set of patterns.

We end this introduction with a short sketch of the structure of this paper. In Section 2 we derive equations (1.4), (1.5), and (1.6), with extra condition (1.7). We also pay some attention to the problem studied by Metzener and Proctor [22] and relate it to our approach. Section 3 is devoted to the derivation and analysis of the stationary problem associated with the nonlocal problem (1.6). The stationary problem associated with the singularly perturbed problem is studied in Sections 4 and 5: In Section 4 we show the existence of (fast) periodic solutions using a Poincaré map and in Section 5 we employ the ideas of geometric singular perturbation theory. We end the paper with a short discussion.

\section{The Derivation of the Equations}

We consider the following model problem:

$$
\frac{\partial \psi}{\partial t}=L_{R, S, T}(\psi)+N(\psi) \quad \text { where } \psi(x, t): \mathbb{R} \times \mathbb{R}^{+} \rightarrow \mathbb{R},
$$

which is a simplification of (1.1) since we restrict ourselves to a one-dimensional problem without a bounded $y$-variable. Furthermore we assume, as in the introduction, that there is a reflection symmetry $x \rightarrow-x$ in (2.1) and that the basic solution $\psi_{0} \equiv 0$. Here, the linear operator depends on three bifurcation-parameters $R, S$, and $T$. The 'eigenvalue' $\mu(k, R)$ as defined in the introduction is in this case equal to the symbol of the linear operator $L_{R, S, T}$ :

$$
L_{R, S, T}\left(e^{i k x}\right)=\mu(k, R ; S, T) e^{i k x} .
$$

We consider this very simple model in order to simplify the derivation of the modulation equation as much as possible. Introducing transversal $y$-dimensions will merely increase 
the technical difficulties. The validity of the Ginzburg-Landau equation (1.2) for systems like (2.1) has been proved in [12].

As in the introduction, we define the neutral curve $\{\operatorname{Re} \mu(k, R)=0\}$. Here, we will study the case that this curve has two minima: $\left(k_{1}, R_{1}\right)$ and $\left(k_{2}, R_{2}\right)$, with $k_{2}<k_{1}$. The neutral curve near $\left(k_{1}, R_{1}\right)$ can be scaled such that

$$
R=1+(k-1)^{2}+\text { h.o.t. }
$$

locally; thus $\left(k_{1}, R_{1}\right)=(1,1)$ (compare with the general expression (1.3)). The two conditional parameters $S$ and $T$ can now be interpreted. By changing $T$, the relative position of $R_{2}$ with respect to $R_{1}=1$ can be adjusted. The relative width of the critical curve, or the band of unstable waves, at $k_{1}=1$ and at $k_{2}$ is changed by $S$. The neutral curve near $\left(k_{2}, R_{2}\right)$ can be written as

$$
R=R_{2}+D\left(k-k_{2}\right)^{2}+\text { h.o.t. }
$$

and thus $D=D(S)$ measures the relative widths of the $(1,1)$ - and $\left(k_{2}, R_{2}\right)$-parabolas.

The object of nonlinear (stability) theory is to describe the nonlinear evolution of the perturbation for $R$ close to the critical value $R_{c}$. If $O\left(R-R_{1}\right) \neq O\left(R-R_{2}\right)$ one derives a single uncoupled Ginzburg-Landau equation (1.2) in the weakly nonlinear stability analysis, either near $(1,1)$ if $1<R_{2}$ or near $\left(k_{2}, R_{2}\right)$ if $1>R_{2}$. Coupling occurs if we assume that

$$
R-1=r \varepsilon^{2}, \quad R-R_{2}=s \varepsilon^{2}, \quad 0<\varepsilon \ll 1 .
$$

This can be seen as follows: One models the perturbation of the basic solution as slow modulations of the critical waves, $e^{i x}$ and $e^{i k_{2} x}$, and their complex conjugates,

$$
\psi(x, t)=\varepsilon A(\xi, \tau) e^{i x}+\varepsilon B(\xi, \tau) e^{i k_{2} x}+c . c .+O\left(\varepsilon^{2}\right),
$$

where $A$ and $B$ are unknown 'amplitudes' of the slow space and time variables $\xi=\varepsilon x$ and $\tau=\varepsilon^{2} t$. The nonlinear terms in (2.1) will generate harmonics of these simple linear waves. Thus, the $\varepsilon^{2}, \varepsilon^{3}$-terms are constructed from a product of the two most unstable waves, $e^{i x}$ and $e^{i k_{2} x}$,

$$
\psi(x, t)=\begin{gathered}
e^{i x}\left[\varepsilon A+\varepsilon^{2} \phi_{12}+\varepsilon^{3} \phi_{13}+\cdots\right] \\
e^{i k_{2} x}\left[\varepsilon B+\varepsilon^{2} \psi_{12}+\varepsilon^{3} \psi_{13}+\cdots\right] \\
\varepsilon^{2} \phi_{02}+\cdots \\
e^{2 i x}\left[\varepsilon^{2} \phi_{22}+\cdots\right] \\
e^{2 i k_{2} x}\left[\varepsilon^{2} \psi_{22}+\cdots\right] \\
e^{i x\left(1+k_{2}\right)}\left[\varepsilon^{2} \Phi_{12}+\cdots\right] \\
e^{i x\left(1-k_{2}\right)}\left[\varepsilon^{2} \Psi_{12}+\cdots\right]
\end{gathered}+\text { c.c. }
$$

Here the $A, B, \phi_{i j}, \psi_{i j}, \Phi_{i j}$, and $\Psi_{i j}$ are functions of $\xi$ and $\tau$ for every $i, j \in \mathbb{N}$. All scalings are classical; see for instance [9]. The validity of this expansion is proven in [10] for the case that there is one minimum.

This expansion is valid as long as there are no low-order resonances between $k_{1}=1$ and $k_{2}$. It is clear that for $k_{2} \neq \frac{1}{2}$ all above interaction terms are different. For $k_{2}=\frac{1}{2}$ some of these terms coincide. As a consequence one has to choose other temporal and 
spatial scales and quadratic terms will appear in the governing evolution equations (see [24] and [31] for physical examples). This is called 'resonance'; we will not discuss this in more detail in this paper. Resonance also occurs for $k_{2}=\frac{1}{3}$ on the $O\left(\varepsilon^{3}\right)$-level. Since the dominant terms of the modulation equations are determined at the $O\left(\varepsilon^{3}\right)$-level there are no other resonant values of $k_{2}$.

The idea behind the derivation of the modulation equation is simple: One substitutes the expansion for $\psi$ into (2.1), and one expands and then gathers terms of the form $\varepsilon^{a} e^{i x\left(b_{1}+b_{2} k_{2}\right)}, a, b_{1}, b_{2} \in \mathbb{N}$. The equations at the $a=2$-level can be solved: The functions in expansion (2.6) can all be expressed in terms of $A$ and $B$. The solvability conditions for $\phi_{13}$ and $\psi_{13}$ at the levels $a=3, b_{1}=1, b_{2}=0$, and $a=3, b_{1}=0$, $b_{2}=1$ yield, after some trivial rescalings, the coupled system (1.4) for $A$ and $B$ given in the introduction.

In this paper we study the situation in which the local parabola near $\left(k_{2}, R_{2}\right),(2.4)$, is very narrow with respect to (2.3) (see the introduction and Figure 1); thus we assume that

$$
D=\frac{1}{\delta^{2}} \quad \text { with } \quad 0<\delta \ll 1
$$

This automatically yields the singularly perturbed system (1.5) given in the introduction. The appearance of the singular term $\frac{1}{\delta^{2}} \frac{\partial^{2} B}{\partial \xi^{2}}$ can also be understood directly from the derivational point of view: The width of the $\left(k_{2}, R_{2}\right)$-parabola at $R=R_{2}+O\left(\varepsilon^{2}\right)$ is $O(\varepsilon \delta)$, so the natural spatial scale associated with the $B$-mode is $\xi_{2}=\varepsilon \delta x=\delta \xi$. Thus, $B$ evolves on a slow spatial scale, compared to $A$. Therefore, it is natural to assume a 'Landau ansatz' for $B: B=B(\tau)$, that is, $B$ is independent of $\xi$, as has been done in [22]. This Ansatz means that we approximate the $\left(k_{2}, R_{2}\right)$-parabola by a line. Repeating the above derivation process we see that this Landau reduction has no influence on the equation for $A$. However, the equation for $\psi_{13}$ (see expansion (2.6)) now reads

$$
L_{R, S, T}\left(\psi_{13} e^{i k_{2} x}\right)=\left[\alpha_{1} \frac{\partial B}{\partial \tau}-\left(\alpha_{2} B+\alpha_{3} B|B|^{2}+\alpha_{4} B|A|^{2}\right)\right] e^{i k_{2} x},
$$

where the $\alpha_{1}, \ldots, \alpha_{4}$ are the nonscaled counterparts of the constants in (1.4). This equation can be written as

$$
L_{R, S, T}\left(\psi_{13} e^{i k_{2} x}\right) e^{-i k_{2} x}=f(\xi, \tau)+g(\tau),
$$

where $f(\xi, \tau)=-\alpha_{4} B|A|^{2}$ is the only term which depends on $\xi$. We define the averages

$$
\begin{aligned}
\overline{\psi_{13}}(\tau) & =\lim _{M \rightarrow \infty} \frac{1}{2 M} \int_{-M}^{M} \psi_{13}(\xi, \tau) d \xi, \\
\bar{f}(\tau) & =\lim _{M \rightarrow \infty} \frac{1}{2 M} \int_{-M}^{M} f(\xi, \tau) d \xi,
\end{aligned}
$$

and separate $f$ and $\psi_{13}$ into a part which only depends on $\tau$ and a part which still depends on both $\xi$ and $\tau$ :

$$
\begin{aligned}
\psi_{13}(\xi, \tau) & =\overline{\psi_{13}}(\tau)+\Psi(\xi, \tau) \\
f(\xi, \tau) & =\bar{f}(\tau)+F(\xi, \tau) .
\end{aligned}
$$


Note that although we of course assume that $\psi_{13}$ and $f$ are bounded on $\mathbb{R}$, these averages do not automatically exist for all $\psi_{13}$ and $f$. However, we shall see that $\overline{\psi_{13}}$ and $\bar{f}$ exist for the functions studied here (see Section 3.2). We substitute these expressions into (2.9) and obtain

$$
L_{R, S, T}\left(\overline{\psi_{13}} e^{i k_{2} x}\right) e^{-i k_{2} x}+L_{R, S, T}\left(\Psi e^{i k_{2} x}\right) e^{-i k_{2} x}=(\bar{f}+g)+F(\xi, \tau)
$$

Because $\overline{\psi_{13}}$ only depends on $\tau$, the first term of this expression can be calculated as

$$
L_{R, S, T}\left(\overline{\psi_{13}} e^{i k_{2} x}\right) e^{-i k_{2} x}=\mu\left(k_{2}, R_{2}\right) \overline{\psi_{13}}=0 .
$$

Thus we get

$$
L_{R, S, T}\left(\Psi e^{i k_{2} x}\right) e^{-i k_{2} x}=(\bar{f}+g)+F .
$$

Taking the above-defined average on both sides leads to the following solvability equation:

$$
\alpha_{1} \frac{\partial B}{\partial \tau}=\alpha_{2} B+\alpha_{3} B|B|^{2}+\alpha_{4} B \lim _{M \rightarrow \infty} \frac{1}{2 M} \int_{-M}^{M}|A|^{2} d \xi .
$$

This equation follows from (2.11); however, it is not a sufficient condition to solve (2.11): The equation for $\Psi$ is still left. Writing $\Psi$ and $F$ as (formal) Fourier integrals, we have

$$
\int_{-\infty}^{\infty}\left(\mu\left(k_{2}+\varepsilon K, R\right) \hat{\Psi}(K)-\hat{F}(K)\right) e^{i K \xi} d K=0,
$$

in the sense of distributions. Now we note that $\mu\left(k_{2}+\varepsilon K, R\right)=O\left(\varepsilon^{2}\right)$ if $|K|=O(\delta)$ (since the local neutral $\left(k_{2}, R_{2}\right)$-parabola is only of $O(\delta)$ width). Thus we see that (2.12) cannot be solved for a bounded $O(1)$ solution $\Psi$ if $\hat{F}(K) \neq O\left(\varepsilon^{2}\right)$ for $|K|=O(\delta)$. This yields a second solvability condition on $F=f-\bar{f}=\alpha_{4} B|A|^{2}-\bar{f}$,

$$
f_{2} B \int_{-\infty}^{\infty}\left(|A|^{2}-\lim _{M \rightarrow \infty} \frac{1}{2 M} \int_{-M}^{M}|A|^{2} d \hat{\xi}\right) e^{i K \xi} d \xi=0+\text { h.o.t. }
$$

for $|K| \leq O(\delta)$. Observe that (2.11) can now be solved. After rescaling, the above analysis leads to the nonlocal system (1.6) given in the introduction, where again $t_{1}, t_{2}$, $r, s, c_{1}$, and $c_{2}$ have exactly the same values as in (1.5); extra condition (2.13) coincides with (1.7). In [3] a proof has been given of the asymptotic validity of a Ginzburg-Landau equation with an extra nonlocal term combined with some additional conditions for a certain version of the Poiseuille flow problem. Note that intuitively the relation between the singularly perturbed system and the nonlocal system is quite simple: $\delta$ has become so small that one is forced to assume that $B$ cannot be a function of $\xi$ (at the highest order). This has no influence on the equation for $A_{\tau}$ in (1.5). However, the $B_{\xi \xi}$ has to disappear in the $B_{\tau}$-equation, and one has to eliminate the $\xi$-dependence of the $|A|^{2}$-term.

Remark 2.1. The above derivation of the nonlocal system (1.6) is not completely rigorous. In order to improve this, one should work with the Fourier transform $\hat{\psi}$ of $\psi$, the solution of (2.1), and interpret it as a distribution; see for instance [12] and [3]. 
Remark 2.2. In their analysis of pattern evolution with disparate scales, Metzener and Proctor [22] do not use the relative width of the local parabolas as small parameter, but the second critical wavenumber $k_{2}: k_{2}=\sigma \ll 1=k_{1}$. If this is the case, it is easy to show that the width of the $\left(k_{2}, R_{2}\right)$-parabola must also be small, so the above derivation covers this case. Note that we assume-as in [22]—-that the $\left(k_{2}, R_{2}\right)$-parabola yields a classical Ginzburg-Landau equation if we omit the $A$-mode (see the introduction); in other words, we assume that $\mu\left(0, R_{1}\right)>0$ and $O(1)$ (see [18] for a short discussion). In this remark we sketch the extra complications encountered by taking $\sigma$ small. If one derives the coupled system in this case, one finds that, if $\sigma \gg \varepsilon$,

$$
\left\{\begin{array}{l}
\frac{\partial A}{\partial \tau}=r A+\frac{\partial^{2} A}{\partial \xi^{2}}+A\left(t_{1}|A|^{2}+\frac{\tilde{c}_{1}}{\sigma^{2}}|B|^{2}\right), \\
\frac{\partial B}{\partial \tau}=s B+\frac{d}{\sigma^{2}} \frac{\partial^{2} B}{\partial \xi^{2}}+B\left(t_{2}|B|^{2}+\frac{\tilde{c}_{2}}{\sigma^{2}}|A|^{2}\right) .
\end{array}\right.
$$

Thus, the coupling terms $A|B|^{2}$ and $B|A|^{2}$ must also be large, $O\left(\frac{1}{\sigma^{2}}\right)$. This is due to the fact that the terms $e^{i(1 \pm \sigma) x}$ in the expansion of $\psi$ (see (2.6)) are now close to the critical wave $e^{i x}$. Thus, solving the equations for $\Phi_{12}, \Psi_{12},(2.6)$, yields terms like $\frac{A B, A \bar{B}}{\mu(1 \pm \sigma, 1)}=O\left(\frac{1}{\sigma^{2}}\right)$. This case is much harder to study than the case above. Therefore, we focus in this paper on the assumption that $k_{2}=O(1), D=\frac{1}{\delta^{2}}, \delta \ll 1$.

Moreover, one encounters many other complications in deriving and studying the appropriate equations as the relative magnitudes of $\varepsilon$ and $\sigma$ change. For instance, for $\varepsilon=O(\sigma)$ the system becomes (at leading order)

$$
\left\{\begin{array}{l}
\frac{\partial A}{\partial \tau}=r A+\frac{\partial^{2} A}{\partial \xi^{2}}+c A\left(B e^{i L \xi}+\bar{B} e^{-i L \xi}\right) \\
\frac{\partial B}{\partial \tau}=s B+a_{1} \lim _{M \rightarrow \infty} \frac{1}{2 M} \int_{-M}^{M}|A|^{2} e^{-i L \xi} d \xi
\end{array}\right.
$$

where $k_{2}=\sigma=\varepsilon L$. In the derivation of these last equations the magnitude of the perturbation had to be taken of order $\varepsilon^{2}$ instead of $\varepsilon$. Solutions of the above system can be found explicitly and it can be checked that these solutions are unstable. From this it follows that although one first has to take the perturbations of magnitude $O\left(\varepsilon^{2}\right)$, they will grow to a magnitude of $O(\varepsilon)$. This leads to a rather complicated system. The above example (2.15) is just included to indicate the complications caused by decreasing $\sigma$ further. Deriving and analysing the full set of equations for every possible combination of the magnitude of $\varepsilon$ relative to that of $\sigma$ is a task we will not pursue in this paper. We refer to [29] in which such a complete nonlinear stability analysis has been performed for what is, in a sense, a simpler case: a weakly, periodically driven, system. There, five essentially different types of modulation equations have been derived.

In [22] a different system has been proposed to describe the weakly nonlinear evolution of patterns at 'disparate scales.' This system is in some sense a combination of (1.6) and (2.15),

$$
\left\{\begin{array}{l}
\frac{\partial A}{\partial \tau}=\mu A-|A|^{2} A+\frac{\partial^{2} A}{\partial X^{2}}+A\left(B e^{i X}+B^{*} e^{-i X}\right) \\
\frac{\partial B}{\partial \tau}=v B-c|B|^{2} B-s \lim _{M \rightarrow \infty} \frac{1}{2 M} \int_{-M}^{M}|A|^{2} e^{-i X} d X
\end{array}\right.
$$

In order to give a foundation to this system one has to assume relations between the parameters of (1.6) and (2.15) and $\varepsilon$ (see [18]): That is the only way to have quadratic and 
cubic terms of the same magnitude. Since these parameters are in principle completely independent of $\varepsilon$ this assumption will be violated in general. Therefore, one will not find this system if one pursues the above-sketched task of deriving all relevant 'generic' equations.

\section{The Nonlocal System}

From now on we focus on the analysis of nonlocal system (1.6) and singularly perturbed system (1.5). We will study and compare the solutions of these systems. System (1.6) can be considered as a limit of (1.5); thus we expect that some classes of solutions represent similar patterns in the underlying basic system. The most simple solutions are the stationary solutions, which we will study here. The stationary solutions are certainly of a physical relevance, for instance in the convection context where stable stationary patterns exist (see Section 6). We first determine the ODE associated with the stationary solutions of (1.6),

$$
\left\{\begin{aligned}
\frac{\partial^{2} A}{\partial \xi^{2}} & =-r A-A\left(t_{1}|A|^{2}+c_{1}|B|^{2}\right) \\
0 & =B\left(s+t_{2}|B|^{2}+c_{2} \lim _{M \rightarrow \infty} \frac{1}{2 M} \int_{-M}^{M}|A|^{2} d \xi\right) .
\end{aligned}\right.
$$

Thus, there are two possible values for $B$ :

1. $B=0$,

2. $|B|^{2}=\frac{-s}{t_{2}}+\frac{-c_{2}}{t_{2}} C(A)$, where $C(A)=\lim _{M \rightarrow \infty} \frac{1}{2 M} \int_{-M}^{M}|A|^{2} d \xi$.

The first case leads to the stationary uncoupled Ginzburg-Landau equation for $A$ (which is integrable; see below and [4]). The second case leads to the following equation for $A$ :

$$
\frac{\partial^{2} A}{\partial \xi^{2}}=\left(-r+\frac{c_{1}}{t_{2}}\left(s+c_{2} C(A)\right)\right) A-t_{1} A|A|^{2} .
$$

To reduce the number of unknown variables we introduce polar coordinates. Let

$$
A=\rho_{1} e^{i \theta_{1}}
$$

where $\rho_{1}$ and $\theta_{1}$ depend on $\xi$. We insert these expressions into the equation for $A$, separate the real and complex parts and find

$$
\left\{\begin{aligned}
\frac{\partial^{2} \rho_{1}}{\partial \xi^{2}}-\rho_{1}\left(\frac{\partial \theta_{1}}{\partial \xi}\right)^{2} & =\left(-r+\frac{c_{1}}{t_{2}}\left(s+c_{2} C\left(\rho_{1}\right)\right)\right) \rho_{1}-t_{1} \rho_{1}^{3}, \\
2 \frac{\partial \rho_{1}}{\partial \xi} \frac{\partial \theta_{1}}{\partial \xi}+\rho_{1} \frac{\partial^{2} \theta_{1}}{\partial \xi^{2}} & =0 .
\end{aligned}\right.
$$

Because

$$
\frac{1}{\rho_{1}} \frac{\partial}{\partial \xi}\left(\rho_{1}^{2} \frac{\partial \theta_{1}}{\partial \xi}\right)=2 \frac{\partial \rho_{1}}{\partial \xi} \frac{\partial \theta_{1}}{\partial \xi}+\rho_{1} \frac{\partial^{2} \theta_{1}}{\partial \xi^{2}}
$$

we find that

$$
\frac{\partial}{\partial \xi}\left(\rho_{1}^{2} \frac{\partial \theta_{1}}{\partial \xi}\right)=0
$$


and therefore introduce the integral $\Omega_{1}$,

$$
\rho_{1}^{2} \frac{\partial \theta_{1}}{\partial \xi}=\Omega_{1}
$$

Substituting this into (3.3) leads to

$$
\frac{\partial^{2} \rho_{1}}{\partial \xi^{2}}=\left(-r+\frac{c_{1}}{t_{2}}\left(s+c_{2} C\left(\rho_{1}\right)\right)\right) \rho_{1}-t_{1} \rho_{1}^{3}+\frac{\Omega_{1}^{2}}{\rho_{1}^{3}} .
$$

To simplify the calculations, we set in system (3.4) $r=1, t_{1}=-1, t_{2}=-1$. Note that this can be obtained by straightforward rescalings with additional assumptions on the signs of $r, t_{1}, t_{2}$. These assumptions are so that the equation is directly related to the single Ginzburg-Landau equation mostly studied in the literature. Furthermore we assume that $\Omega_{1}=0$. Taking $\Omega_{1}=0$ simplifies the analysis. However, for $\Omega_{1} \neq 0$ the analysis is in essence the same. Because $\Omega_{1}=\rho_{1}^{2} \frac{\partial \theta_{1}}{\partial \xi}, \Omega_{1}=0$ implies that $\frac{\partial \theta_{1}}{\partial \xi}=0$. This yields that $\theta_{1}$ does not depend on $\xi$; thus $\theta_{1}$ is a constant. Therefore, due to the phase shift invariance in (1.6), one can say that we restrict ourselves to studying real solutions of this system. We refer to [4] for a detailed discussion of the relation between $\Omega=0$ and $\Omega \neq 0$ in the single real Ginzburg-Landau case.

\subsection{Stationary Solutions of the Nonlocal Equations}

We introduce $x=\rho_{1}$ in (3.4) with $\Omega_{1}=0, r=1, t_{1}=-1$, and $t_{2}=-1$,

$$
\ddot{x}=-\left(1+c_{1}\left(s+c_{2} C(x)\right)\right) x+x^{3} \quad \text { with } \quad C(x)=\lim _{M \rightarrow \infty} \frac{1}{2 M} \int_{-M}^{M} x^{2} d t,
$$

where the dot means differentiating with respect to 'time' $t=\xi$. We are only interested in bounded solutions of these equations since $A$, where $|A|=\rho_{1}=x$, must remain bounded. Moreover $C(x)$ is only defined for bounded $x$. First we set $C(x)=\mathcal{C}$, where $\mathcal{C}$ is a fixed constant. System (3.5) then becomes

$$
\ddot{x}=-a x+x^{3} \quad \text { where } \quad a=1+c_{1}\left(s+c_{2} \mathcal{C}\right) .
$$

We will describe the phase portrait of this equation in some detail, because later on we will come across this equation again. For $a<0$, (3.6) has only one critical point, $(0,0)$, which is a saddle point. This shows that there are no bounded solutions, except for the trivial critical point. For $a>0$, the system has three critical points: $(0,0)$ and $( \pm \sqrt{a}, 0)$. In this case $(0,0)$ is a centre point and $( \pm \sqrt{a}, 0)$ are saddle points. There exist two heteroclinic connections between the two saddle points. Inside this heteroclinic loop there are bounded periodic solutions and outside all orbits are unbounded. This means that bounded solutions of (3.6) will always lie inside the heteroclinic cycle formed by the two connections. These solutions are periodic (see Figure 2). System (3.6) is integrable with integral or energy $k$ :

$$
k=\frac{1}{2} y^{2}+\frac{1}{2} a x^{2}-\frac{1}{4} x^{4}, \quad \text { where } y=\dot{x} .
$$




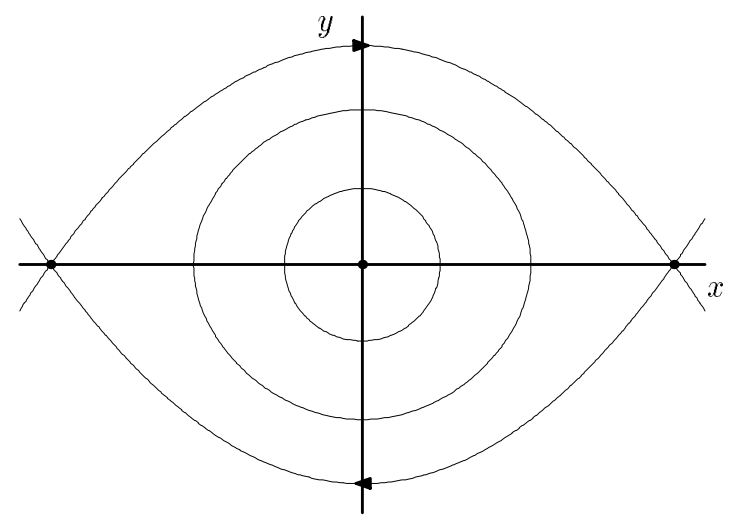

Fig. 2. The phase portrait for the equation $\ddot{x}=-a x+x^{3}$ where $a>0$.

It is possible to determine $C(x)$ explicitly for a periodic solution of (3.6), with period $T_{0}$. Note that

$$
C(x)=\lim _{M \rightarrow \infty} \frac{2 M}{2 M T_{0}} \int_{0}^{T_{0}} x^{2} d t=\frac{1}{T_{0}} \int_{0}^{T_{0}} x^{2} d t
$$

for a periodic solution $x$. Hence

$$
C(x)=\frac{\int_{0}^{T_{0}} x^{2} d t}{\int_{0}^{T_{0}} d t}=\frac{\int_{-x_{1}}^{x_{1}} \frac{x^{2}}{G(x, k)} d x}{\int_{-x_{1}}^{x_{1}} \frac{1}{G(x, k)} d x},
$$

by changing variables, where $G(x, k)=\sqrt{2 k-a x^{2}+\frac{1}{2} x^{4}}$. Here $-\sqrt{a}<-x_{1}<0<$ $x_{1}<\sqrt{a}$ are the intersection points of the solution $x$, on the energy level set $k$, with $y=0$. Introduce $X=x^{2}$ and $X_{1}=x_{1}^{2}$, then

$$
C(x)=\frac{T_{1}(k)}{T_{0}(k)}, \quad \text { where } T_{i}(k)=\oint \frac{X^{i} d X}{\sqrt{2 k X-a X^{2}+\frac{1}{2} X^{3}}} .
$$

This is a contour integral in the complex plane around the interval $\left[0, X_{1}\right]$ on the real axis. We define

$$
\chi(k)=\frac{T_{1}(k)}{T_{0}(k)} .
$$

Because $x$ is a periodic solution which lies inside the heteroclinic cycle, the $k$-value of $x$ lies between 0 and $\frac{1}{4} a^{2}$. Below we will show that $\chi$ is a monotonic function of $k$, so we can conclude that $0<\chi<a$ (since $\chi(0)=0$ and $\lim _{k \uparrow \frac{1}{4} a^{2}} \chi(k)=a$ ). Although this result is a special case of a more general result proved in [4], we will sketch the derivation of the monotonicity result: $\chi(k)$ is an important quantity which will also appear in subsequent sections. Note that

$$
\frac{\partial \chi}{\partial k}=\frac{\partial}{\partial k} \frac{T_{1}}{T_{0}}=\frac{T_{0} \frac{\partial T_{1}}{\partial k}-T_{1} \frac{\partial T_{0}}{\partial k}}{T_{0}^{2}} .
$$


We define $G_{1}(k, X)=\sqrt{2 k X-a X^{2}+\frac{1}{2} X^{3}}$ and define

$$
J_{i}(k)=\oint \frac{X^{i} d X}{\left(G_{1}(k, X)\right)^{3}},
$$

which yields that

$$
\frac{\partial \chi}{\partial k}=\frac{-T_{0} J_{2}+T_{1} J_{1}}{T_{0}^{2}} .
$$

Rewriting $T_{i}(k)$ into these $J_{i}(k)$ 's leads to the following relation for every $i \geq 0$ :

$$
T_{i}=2 k J_{i+1}-a J_{i+2}+\frac{1}{2} J_{i+3}
$$

Since

$$
\oint \frac{d}{d X} \frac{X^{i} d X}{\left(G_{1}(k, X)\right)}=0
$$

for every $i \geq 1$, we also find

$$
k(2 i-1) J_{i}+(1-i) a J_{i+1}+\left(\frac{1}{2} i-\frac{3}{4}\right) J_{i+2}=0 .
$$

Setting in (3.13) $i=0,1$ and in (3.14) $i=1,2$ leads to a system of four equations from which $J_{1}$ and $J_{2}$ can be solved. Substitution of these expressions into (3.12) gives

$$
\frac{\partial \chi}{\partial k}=\frac{1}{4 k\left(a^{2}-4 k\right)}\left(a \chi^{2}-8 k \chi+4 k a\right) .
$$

We define $f(k)=4 k\left(a^{2}-4 k\right)$ and $P(\chi)=a \chi^{2}-8 k \chi+4 k a$, and see that $f(k)>0$ for $0<k<\frac{1}{4} a^{2}$, which is exactly the interval we are studying. There are no solutions of $P(\chi)=0$ and so, because $a>0, P(\chi)>0$ for every $\chi$. Combining this finally results in $\frac{\partial \chi}{\partial k}>0$. This yields that $0<\chi(k)<a$, where $\chi(k)=0$ corresponds to the centre point $(0,0)$ and $\chi(k)=a$ to the heteroclinic orbit. Since $C(x)=\chi(k)$ one observes that bounded solutions can only exist if $0<C(x)<a$. We can now explicitly solve (3.5): For a given value of $a=1+c_{1}\left(s+c_{2} \mathcal{C}\right)$ in (3.6) we see that the bounded orbits have values of $\mathcal{C}$ ranging from 0 to $a$; thus if

$$
C(x) \in\left(0,1+c_{1}\left(s+c_{2} C(x)\right)\right) \text {, }
$$

we see that one bounded orbit of system (3.6) is selected as the solution of system (3.5). Again $C(x)=0$ corresponds to the selection of the centre point $(0,0)$ and $C(x)=$ $1+c_{1}\left(s+c_{2} C(x)\right)$, i.e., $C(x)=\frac{1+c_{1} s}{1-c_{1} c_{2}}$, to the selection of a heteroclinic orbit. Recall that $|B|^{2}=s+c_{2} C(x)$, which relates the value of $|B|$ to every $C(x)$. Thus, the nonlocal system (1.6) only has bounded stationary solutions $(A(\xi), B)$ with 'average' $C(A)$ if $0<C(A)<1+c_{1}\left(s+c_{2} C(A)\right)$ and $|B|^{2}=s+c_{2} C(A)>0$. 


\subsection{Applying the Solvability Condition}

The two boundaries of the interval given by (3.15) determine, in a sense, the bifurcations at which the periodic solutions of (3.5) (dis)appear. As already noted above, the periodic solution shrinks into a critical point at $C(x)=0$. The other boundary, at $C(x)=\frac{1+c_{1} s}{1-c_{1} c_{2}}$, determines a global bifurcation at which the periodic orbit merges with a heteroclinic cycle of (3.5). From the derivation of the system we also obtained an extra condition (1.7) which the solutions have to satisfy. As can be easily seen, the critical points of the system do satisfy the condition. This is also true for the periodic solutions as long as their period is not too large. This can be seen as follows. Note that (1.7) determines the Fourier transform of the (periodic) function $|A|^{2}-C(A)$. This is of course a discrete spectrum with 'peaks' at $K=\frac{2 \pi n}{T_{0}}, n= \pm 1, \pm 2, \ldots$, where $T_{0}$ is the period of $|A|^{2}$. Observe that there is no peak at $K=0$ due to the subtraction ' $-C(A)$ '. Since $T_{0}$ becomes unbounded as $k \uparrow \frac{1}{4} a^{2}$ (see [4]), we see that the $n= \pm 1$ peaks approach the $K=O(\delta)$ region if $k$ approaches $\frac{1}{4} a^{2}$. In other words, the periodic orbits satisfy the extra condition (1.7) as long as $T_{0} \neq O\left(\frac{1}{\delta}\right)$. The periodic orbits with a very long period do not satisfy (1.7). The same is true for the heteroclinic orbits: They do not satisfy (1.7) (this can be checked by using the explicit expression (5.3) given in Section 5.1). Thus, we conclude that the only stationary solutions described by the Landau reduction are the solutions with $|A|=$ constant or $|A|$ is periodic with $O(1)$ period. All other solutions of system (1.6) do not satisfy solvability condition (1.7).

Furthermore, we note that the 'average' described in Section 2 is defined for all solutions of the stationary problem associated with (1.6).

\section{The Singularly Perturbed System: Periodic Solutions}

In this section we will study the stationary solutions of the singularly perturbed system. The stationary problem associated with the singularly perturbed system reads

$$
\left\{\begin{array}{l}
\frac{\partial^{2} A}{\partial \xi^{2}}=-r A-A\left(t_{1}|A|^{2}+c_{1}|B|^{2}\right), \\
\frac{\partial^{2} B}{\partial \xi^{2}}=\delta^{2}\left(-s B-B\left(t_{2}|B|^{2}+c_{2}|A|^{2}\right)\right) .
\end{array}\right.
$$

As in Section 3, we introduce polar coordinates for $A$ and $B$,

$$
A=\rho_{1} e^{i \theta_{1}}, \quad B=\rho_{2} e^{i \theta_{2}} .
$$

Then the system becomes

$$
\left\{\begin{array}{l}
\frac{\partial^{2} \rho_{1}}{\partial \xi^{2}}=-r \rho_{1}-\rho_{1}\left(t_{1} \rho_{1}^{2}+c_{1} \rho_{2}^{2}\right)+\frac{\Omega_{1}^{2}}{\rho_{1}^{3}} \\
\frac{\partial^{2} \rho_{2}}{\partial \xi^{2}}=\delta^{2}\left(-s \rho_{2}-\rho_{2}\left(t_{2} \rho_{2}^{2}+c_{2} \rho_{1}^{2}\right)+\frac{\tilde{\Omega}_{2}^{2}}{\rho_{2}^{3}}\right),
\end{array}\right.
$$

where $\Omega_{1}$ and $\tilde{\Omega}_{2}$ are integrals, similar to $\Omega_{1}$ in Section 3: $\Omega_{1}=\rho_{1}^{2} \frac{\partial \theta_{1}}{\partial \xi}$ and $\delta \tilde{\Omega}_{2}=\Omega_{2}=$ $\rho_{2}^{2} \frac{\partial \theta_{2}}{\partial \xi}$. Note that $\frac{\partial B}{\partial \xi}=O(\delta)$; thus $\frac{\partial \theta_{2}}{\partial \xi}$ has to be of $O(\delta)$, which implies that $\Omega_{2}$ is $O(\delta)$ : $\Omega_{2}=\delta \tilde{\Omega}_{2}$. We will study the bounded solutions of singularly perturbed system (4.2) 
where, as for the nonlocal system, we set for simplicity $\Omega_{1}=\tilde{\Omega}_{2}=0$ (and $r=1$, $\left.t_{1}=t_{2}=-1\right)$. This implies that $\frac{\partial \theta_{1}}{\partial \xi}=\frac{\partial \theta_{2}}{\partial \xi}=0$ and thus that $\theta_{1}$ and $\theta_{2}$ are constants. Note that the Stokes waves, $(A=$ constant, $B=0)$ and $(A=0, B=$ constant $)$, are on the $\Omega_{1}=\tilde{\Omega}_{2}=0$ level set.

Next we introduce $x=\rho_{1}$ and $y=\frac{\partial \rho_{1}}{\partial \xi}=\dot{x}$ where the dot means differentiation with respect to 'time' $t=\xi$; equivalently we write $z=\rho_{2}$ and $\delta w=\dot{z}$,

$$
\left\{\begin{array}{l}
\dot{x}=y \\
\dot{y}=-x+x\left(x^{2}-c_{1} z^{2}\right) \\
\dot{z}=\delta w \\
\dot{w}=\delta\left(-s z+z\left(z^{2}-c_{2} x^{2}\right)\right)
\end{array}\right.
$$

Thus, $x$ and $y$ can be considered as the fast $(=O(1))$ moving coordinates and $z$ and $w$ as the slow $(=O(\delta))$ coordinates. The system contains a lot of useful symmetries: (4.3) is equivariant under

$$
\begin{aligned}
& \{x \rightarrow-x, y \rightarrow-y\}, \quad\{x \rightarrow-x, t \rightarrow-t, w \rightarrow-w\}, \quad\{x \rightarrow-x, t \rightarrow-t, z \rightarrow-z\}, \\
& \{z \rightarrow-z, w \rightarrow-w\}, \quad\{y \rightarrow-y, t \rightarrow-t, w \rightarrow-w\}, \quad\{y \rightarrow-y, t \rightarrow-t, z \rightarrow-z\} .
\end{aligned}
$$

System (4.3) can be considered as a Hamiltonian system. We introduce the arbitrary rescalings $x=\alpha \tilde{x}, y=\alpha \tilde{y}, z=\beta \tilde{z}$, and $w=\beta \tilde{w}$, for $\alpha, \beta>0$. This gives

$$
\left\{\begin{array}{l}
\dot{\tilde{x}}=\tilde{y} \\
\dot{\tilde{y}}=-\tilde{x}+\tilde{x}\left(\alpha^{2} \tilde{x}^{2}-c_{1} \beta^{2} \tilde{z}^{2}\right), \\
\dot{\tilde{z}}=\delta \tilde{w} \\
\dot{\tilde{w}}=\delta\left(-s \tilde{z}+\tilde{z}\left(\beta^{2} \tilde{z}^{2}-c_{2} \alpha^{2} \tilde{x}^{2}\right)\right) .
\end{array}\right.
$$

The Hamiltonian $H$ which could belong to this system must be of the form

$$
H=\frac{1}{2}\left(\tilde{x}^{2}+\tilde{y}^{2}+\delta s \tilde{z}^{2}+\delta \tilde{w}^{2}\right)-\frac{1}{4} \alpha^{2} \tilde{x}^{4}-\frac{1}{4} \beta^{2} \tilde{z}^{4}+m \tilde{x}^{2} \tilde{z}^{2}
$$

where $m$ still has to be determined. This implies that we must impose that $c_{1} \beta^{2}=\delta c_{2} \alpha^{2}$, which yields that

$$
\frac{\beta^{2}}{\alpha^{2}}=\frac{\delta c_{2}}{c_{1}}, \quad \text { when } \operatorname{sign}\left(c_{1}\right)=\operatorname{sign}\left(c_{2}\right) .
$$

Thus, it is possible to rescale (4.3) so that it becomes a Hamiltonian system. This rescaling has to satisfy $\frac{\beta^{2}}{\alpha^{2}}=O(\delta)$, which means that $O(1)$ solutions in the Hamiltonian system correspond to solutions of (4.3) of which either the pair $(x, y)$ or the pair $(z, w)$ (or both) is not $O(1)$. However, amplitude $A$ in (1.5) corresponds to $(x, y)$ and $B$ to $(z, w)$ and both $A$ and $B$ must be $O(1)$, due to the structure of the derivation process. Therefore we will not write (4.3) as a Hamiltonian system, but we will use that the energy is conserved. Note that the expression for the energy contains only even powers of $x, y, z$, and $w$. In the case that $\operatorname{sign}\left(c_{1}\right)=-\operatorname{sign}\left(c_{2}\right)$ it is also possible to rescale (4.3) to a Hamiltonian system. 
Before we study the perturbed system we are interested in the dynamics of the unperturbed system. Setting $\delta=0$ leads to

$$
\left\{\begin{array}{l}
\dot{x}=y \\
\dot{y}=-x+x\left(x^{2}-c_{1} z_{0}^{2}\right)
\end{array}\right.
$$

Here $z=z_{0}$ and $w=w_{0}$ where $z_{0}$ and $w_{0}$ are constants of motion, because setting $\delta=0$ implies that $\dot{z}=0$ and $\dot{w}=0$. The above system can also be written as

$$
\ddot{x}=-\left(1+c_{1} z_{0}^{2}\right) x+x^{3} .
$$

This is the same as equation (3.6) with $a=1+c_{1} z_{0}^{2}$, which we already studied in Section 3.1. Therefore the same results as for (3.6) can be obtained.

Next we consider the critical points of the perturbed system. Here the critical points are given with their characterisation, where the first part is the characterisation in the fast directions and the second in the slow directions (that is, the first pair of eigenvalues is $O(1)$, the second pair $O(\delta))$.

$$
\begin{array}{lll}
(0,0,0,0) & \text { for } s>0 & \text { centre/centre, } \\
& \text { for } s<0 & \text { centre/saddle, } \\
(0,0, \pm \sqrt{s}, 0) & \text { for } s c_{1}+1<0 & \text { saddle/saddle, } \\
& \text { for } s c_{1}+1>0 & \text { centre/saddle, } \\
( \pm 1,0,0,0) & \text { for } c_{2}+s<0 & \text { saddle/saddle, } \\
\left( \pm \sqrt{\frac{c_{1} s+1}{1-c_{1} c_{2}}}, 0, \pm \sqrt{\frac{s+c_{2}}{1-c_{1} c_{2}}}, 0\right) & \text { for } c_{2}+s>0 & \text { saddle/centre, } \\
& \text { for } \frac{2\left(c_{2}+s\right)\left(1+c_{1} c_{2}\right)}{1-c_{1} c_{2}}<0 & \text { saddle/centre, } \\
& \text { for } \frac{2\left(c_{2}+s\right)\left(1+c_{1} c_{2}\right)}{1-c_{1} c_{2}}>0 & \text { saddle/saddle. }
\end{array}
$$

These critical points only exist when the expressions under the square root are positive. So the second two critical points only exist for $s>0$ and the last four critical points only exist for $\frac{c_{1} s+1}{1-c_{1} c_{2}}>0$ and $\frac{s+c_{2}}{1-c_{1} c_{2}}>0$. These critical points give rise to solutions of the original system. The first critical point gives the trivial solution, the second gives the Stokes wave $A=0$ and $B \pm \sqrt{s} e^{i \theta_{2}}$ where $\theta_{2}$ is a constant; the third gives another Stokes wave $A= \pm e^{i \theta_{1}}$ and $B=0$ with $\theta_{1}$ a constant. The last four critical points correspond to the mixed patterns $A \equiv \pm \sqrt{\frac{c_{1} s+1}{1-c_{1} c_{2}}} e^{i \theta_{1}}$ and $B \equiv \pm \sqrt{\frac{s+c_{2}}{1-c_{1} c_{2}}} e^{i \theta_{2}}$ with $\theta_{1}$ and $\theta_{2}$ constants. The heteroclinic and homoclinic orbits of Section 5 will have their origins and destinies at one or two of these four points.

\subsection{Periodic Solutions}

The singularly perturbed system possesses two time scales: $(x, y)$ are the fast variables and $(z, w)$ are the slow variables. In the nonlocal system there is no slow behaviour. When studying behaviour which is dominated by the fast field in the singularly perturbed system, we have to take $\dot{z}$ and $\dot{w}$ almost 0 , which implies that $B$ is 'almost' independent of $\xi$. Recall that this is exactly the condition which is imposed on $B$ when deriving the nonlocal system. Therefore we expect to observe approximately the same behaviour for solutions which remain in the fast field of the singularly perturbed system as for the 
solutions of the nonlocal system. In this subsection we will study the solutions of (4.3) which are dominated by the fast field. We will focus on the periodic solutions. We expect to find approximately the same existence conditions (and solutions) as for the nonlocal system. In the next section we will study solutions to (4.3) which consist of fast and slow parts. These solutions cannot exist in the nonlocal system.

We will construct solutions to (4.3) by using the fact that the unperturbed limit $\delta=0$ is completely integrable:

$$
\left\{\begin{array}{l}
\dot{x}=y, \\
\dot{y}=-x+x\left(x^{2}-c_{1} z^{2}\right) \\
\dot{z}=0 \\
\dot{w}=0
\end{array}\right.
$$

with the three integrals

$$
k=\frac{1}{2}\left(y^{2}+x^{2}-\frac{1}{2} x^{4}+c_{1} x^{2} z^{2}\right), \quad k_{1}=z, \quad k_{2}=w .
$$

The behaviour of fast periodic solutions of (4.3) is dominated by the unperturbed system, since the solutions to (4.3) remain $O(\delta)$ close to solutions of (4.8) for $O(1)$ time. Thus, a fast periodic solution to (4.3) will be $O(\delta)$ close to a periodic solution of (4.8): We can study the existence of periodic solutions of the singularly perturbed system by constructing and approximating a Poincaré map which measures the changes in the $k, z$, and $w$-values for a solution of the perturbed system. This Poincaré mapping is defined as follows:

$$
\begin{aligned}
P\left(k, k_{1}, k_{2}\right) & =\left(k+\Delta K\left(k, k_{1}, k_{2}\right), k_{1}+\Delta K_{1}\left(k, k_{1}, k_{2}\right), k_{2}+\Delta K_{2}\left(k, k_{1}, k_{2}\right)\right) \\
& =\left(k+\Delta K(k, z, w), z+\Delta K_{1}(k, z, w), w+\Delta K_{2}(k, z, w)\right) .
\end{aligned}
$$

The quantities $\Delta K\left(k_{0}, z_{0}, w_{0}\right), \Delta K_{1}\left(k_{0}, z_{0}, w_{0}\right)$, and $\Delta K_{2}\left(k_{0}, z_{0}, w_{0}\right)$ measure the accumulated change in the slow variables $k, z$, and $w$ from a solution of the perturbed system. Due to reasons which will become clear later on we define the Poincaré map in a somewhat nonstandard way. The Poincaré map is defined by a solution which consists of two parts which are joined together. The first part starts on the cross section $\{x=0, y>0\}$, travels forwards in time and ends by intersecting the cross section $\{x=0, y<0\}$. The time it takes this solution to intersect with the cross section $\{x=0, y<0\}$ is denoted by $T_{\delta}$. The other part travels backwards in time and ends by intersecting $\{x=0, y<0\}$ (see Figure 3). The time it takes this solution to intersect with $\{x=0, y<0\}$ is denoted by $T_{-\delta}$. Here $\left(k_{0}, z_{0}, w_{0}\right)$ is the initial value of these solutions; hence $k_{0}$ is so that $x_{0}=0$ and $y_{0}>0$. The accumulated change of the integral $k$ over this orbit is given by

$$
\Delta K\left(k_{0}, z_{0}, w_{0}\right)=\int_{-T_{-\delta}}^{T_{\delta}} \dot{k}\left(x_{\delta}, y_{\delta}, z_{\delta}, w_{\delta}\right) d t,
$$

where $\left(x_{\delta}(t), y_{\delta}(t), z_{\delta}(t), w_{\delta}(t)\right)$ is the above-constructed solution of the perturbed system. The quantities $\Delta K_{1}$ and $\Delta K_{2}$ can be expressed in the same way. Substituting the expression for $k,(4.9)$, gives

$$
\Delta K\left(k_{0}, z_{0}, w_{0}\right)=\delta \int_{-T_{-\delta}}^{T_{\delta}} c_{1} x_{\delta}^{2} w_{\delta} z_{\delta} d t
$$




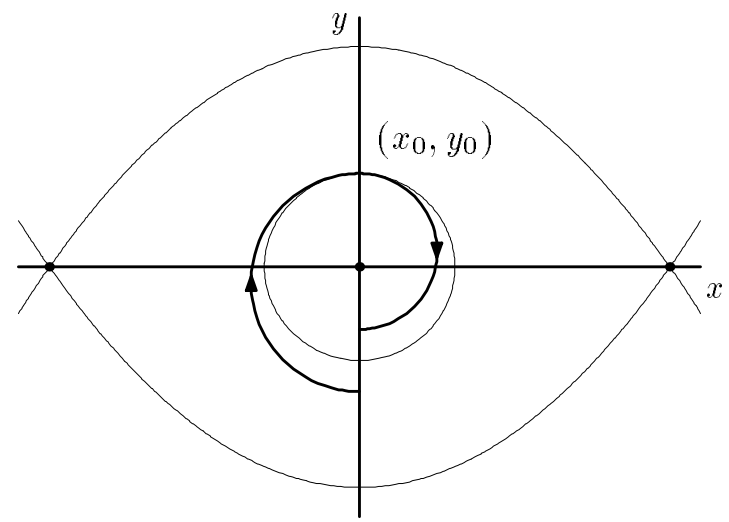

Fig. 3. The construction of the Poincaré map. The thin lines represent solutions to the unperturbed problem (4.6) in the plane $\left\{z=z_{0}, w=w_{0}\right\}$. The thick line is the projection of a solution of (4.1) on the plane $\left\{z=z_{0}, w=\right.$ $\left.w_{0}\right\}$.

The solution $\left(x_{\delta}(t), y_{\delta}(t), z_{\delta}(t), w_{\delta}(t)\right)$ can be approximated with $O(\delta)$-error by the solution $\left(x_{0}(t), y_{0}(t), z_{0}, w_{0}\right)$ of the unperturbed system with energy $k=k_{0}$ which starts on the cross section $\{x=0, y<0\}$ with the same initial data as $\left(x_{\delta}(t), y_{\delta}(t), z_{\delta}(t), w_{\delta}(t)\right)$; $T_{ \pm \delta}$ can be approximated by $\pm \frac{1}{2} T_{0}$, the period of the solution $\left(x_{0}(t), y_{0}(t), z_{0}, w_{0}\right)$. Therefore

$$
\Delta K\left(k_{0}, z_{0}, w_{0}\right)=\delta \int_{-\frac{1}{2} T_{0}}^{\frac{1}{2} T_{0}} c_{1} x_{0}^{2} w_{0} z_{0} d t+O\left(\delta^{2}\right) .
$$

We define $G(k, z, x)=\sqrt{2 k-x^{2}+\frac{1}{2} x^{4}-c_{1} x^{2} z^{2}}$. Note that this is nearly the same function as defined in Section 3.1. Transforming the coordinates leads to

$$
\Delta K\left(k_{0}, z_{0}, w_{0}\right)=2 \delta c_{1} w_{0} z_{0} \int_{-x_{1}}^{x_{1}} \frac{x^{2} d x}{G\left(k_{0}, z_{0}, x\right)}+O\left(\delta^{2}\right) .
$$

Here $-\sqrt{1+c_{1} z_{0}^{2}}<-x_{1}<0<x_{1}<\sqrt{1+c_{1} z_{0}^{2}}$ are the intersection points with $y=0$ of the solution with $k=k_{0}$. We set $X=x^{2}$ and $X_{1}=x_{1}^{2}$; then,

$$
\Delta K\left(k_{0}, z_{0}, w_{0}\right)=\delta c_{1} w_{0} z_{0} T_{1}\left(k_{0}, z_{0}\right)+O\left(\delta^{2}\right),
$$

where

$$
T_{i}(k, z)=\oint \frac{X^{i} d X}{\sqrt{2 k X-X^{2}+\frac{1}{2} X^{3}-c_{1} X^{2} z^{2}}} .
$$

This contour integral around the interval $\left[0, X_{1}\right]$ is again very similar to the one defined in Section 3.1. In the same way we obtain

$$
\begin{aligned}
& \Delta K_{1}\left(k_{0}, z_{0}, w_{0}\right)=\delta w_{0} T_{0}\left(k_{0}, z_{0}\right)+O\left(\delta^{2}\right), \\
& \Delta K_{2}\left(k_{0}, z_{0}, w_{0}\right)=-\delta z_{0}\left(\left(s-z_{0}^{2}\right) T_{0}\left(k_{0}, z_{0}\right)+c_{2} T_{1}\left(k_{0}, z_{0}\right)\right)+O\left(\delta^{2}\right) .
\end{aligned}
$$


A solution is periodic if $\Delta K=\Delta K_{1}=\Delta K_{2}=0$. Since $T_{0}(k, z)$ and $T_{1}(k, z)$ are strictly positive, this yields

$$
\begin{aligned}
c_{1} w_{0} z_{0}+O(\delta) & =0 \\
w_{0}+O(\delta) & =0 \\
z_{0}\left(\left(s-z_{0}^{2}\right) T_{0}\left(k_{0}, z_{0}\right)+c_{2} T_{1}\left(k_{0}, z_{0}\right)\right)+O(\delta) & =0 .
\end{aligned}
$$

This seems to lead to two possibilities:

1. $z_{0}=O(\delta)$ and $w_{0}=O(\delta)$,

2. $w_{0}=O(\delta)$ and $\left(s-z_{0}^{2}\right) T_{0}\left(k_{0}, z_{0}\right)+c_{2} T_{1}\left(k_{0}, z_{0}\right)=O(\delta)$.

However, (4.13) is a singular system in the limit $\delta \rightarrow 0$. Thus we cannot solve this system for $\delta=0$ by applying the implicit function theorem and concluding that there is a solution to the perturbed system $O(\delta)$ near the $\delta=0$ solution. On the contrary: one must expect that the solution of the full problem will be much more complicated. Nevertheless, we will now show that the above two 'singular' solutions are correct.

Approximating the solution of the perturbed system by a solution of the unperturbed system throws away too much of the dynamics of the system; thus we have to look at the expressions without approximating the solution. The exact expressions for $\Delta K, \Delta K_{1}$, and $\Delta K_{2}$ are

$$
\begin{aligned}
\Delta K\left(k_{0}, z_{0}, w_{0}\right) & =\delta \int_{-T_{-}}^{T_{\delta}} c_{1} x_{\delta}^{2} w_{\delta} z_{\delta} d t \\
\Delta K_{1}\left(k_{0}, z_{0}, w_{0}\right) & =\delta \int_{-T_{-\delta}}^{T_{\delta}} w_{\delta} d t \\
\Delta K_{2}\left(k_{0}, z_{0}, w_{0}\right) & =\delta \int_{-T_{-\delta}}^{T_{\delta}}\left(-s z_{\delta}+z_{\delta}^{3}-c_{2} x_{\delta}^{2} z_{\delta}\right) d t .
\end{aligned}
$$

We note that $w_{\delta}(t) \equiv 0$ and $z_{\delta}(t) \equiv 0$ if $z_{0}=w_{0}=0$. Thus $\Delta K\left(k_{0}, 0,0\right)=$ $\Delta K_{1}\left(k_{0}, 0,0\right)=\Delta K_{2}\left(k_{0}, 0,0\right)=0$. Therefore periodic solutions exist. This can also be seen by noting that if $z=w=0$, the dynamics of (4.3) are described by

$$
\ddot{x}=-x+x^{3} .
$$

This leads to periodic solutions in the $(z, w)=(0,0)$-plane. These solutions are also solutions of the unperturbed system.

We now consider the second possible solution to (4.13). On the cross section we have $x_{0}=x(0)=0$. Let's again consider $w_{0}=w(0)=0$; thus $\dot{z}(0)=0$. It can be shown for a solution $\left(x_{\delta}, y_{\delta}, z_{\delta}, w_{\delta}\right)$ of (4.3) with these initial conditions that $x_{\delta}(t)$ (resp. $\left.z_{\delta}(t)\right)$ is an odd (resp. even) function of $t$. This can be done by inductively checking that $x(0)=0$ and $\dot{z}(0)=0$ in (4.3) yields that $x^{(2 n)}(0)=0, z^{(2 n+1)}(0)=0$ for every $n$. From the fact that $x$ is odd it follows that $T_{-\delta}=T_{\delta}$. Because $z$ is even, $w=\dot{z}$ is odd. Thus

$$
\Delta K\left(k_{0}, z_{0}, w_{0}\right)=\delta \int_{-\frac{1}{2} T_{\delta}}^{\frac{1}{2} T_{\delta}} c_{1} x_{\delta}^{2} w_{\delta} z_{\delta} d t \equiv 0,
$$


since $x_{\delta}^{2} w_{\delta} z_{\delta}$ is an odd function of $t$. Analogously, since $w_{\delta}$ is odd,

$$
\Delta K_{1}\left(k_{0}, z_{0}, w_{0}\right) \equiv 0 .
$$

We still have to use the solution of the unperturbed system to approximate $\Delta K_{2}$; see (4.12). Thus, a periodic solution with initial data $x_{0}=z_{0}=0$ must satisfy

$$
z_{0}=0 \quad \text { or } \quad\left(z_{0}^{2}-s\right) T_{0}\left(k_{0}, z_{0}\right)-c_{2} T_{1}\left(k_{0}, z_{0}\right)=0 .
$$

This is equivalent to $\chi\left(k_{0}, z_{0}\right)=\frac{z_{0}^{2}-s}{c_{2}}$ where $\chi$ is in essence the function defined in (3.11). In Section 3.1 we showed that $\chi \in[0, a]$, where $a$ in (3.6) corresponds to $1+c_{1} z^{2}$ in (4.7), and that $\chi$ is a monotonically increasing function of $k$ from 0 to $a=1+c_{1} z^{2}$. Since $s, c_{1}$, and $c_{2}$ are known from the equations, an interval for $z_{0}$ can be determined so that $\chi\left(k_{0}, z_{0}\right)=\frac{z_{0}^{2}-s}{c_{2}}$ holds,

$$
0 \leq \frac{z_{0}^{2}-s}{c_{2}} \leq 1+c_{1} z_{0}^{2}
$$

As in the case for the existence of solutions of the nonlocal system, there are, for different values of the coefficients, different intervals for $z_{0}$ where periodic solutions exist.

\subsection{The Relation with the Nonlocal System}

Since we expected similar conditions for the existence of periodic solutions for the singularly perturbed system as for solutions of the nonlocal system, we are now going to compare the conditions for this existence. Recall that in the nonlocal system bounded solutions exist for $B=0$ and for $|B|^{2}=s+c_{2} C(x)$ where $C(x) \in\left(0,1+c_{1}(s+\right.$ $\left.\left.c_{2} C(x)\right)\right)$. In the singularly perturbed system there exist periodic solutions for $z=0$ and for $z$ with $0 \leq \frac{z^{2}-s}{c_{2}} \leq 1+c_{1} z^{2}$. In Section 4 we set $B=\rho_{2} e^{i \theta_{2}}$ and we introduced $z$ by $\rho_{2}=z$. This implies that $B=0$ is the same as $z=0$. Thus the first possibilities for the existence of the periodic solutions coincide, which of course is not surprising since the nonlocal system (1.6) and the singularly perturbed system (1.5) are exactly the same for the subcase $B=0$. The other two cases are somewhat more difficult to compare but finally these appear to be essentially the same. Since $|B|=z$ in the singularly perturbed case and $|B|^{2}=s+c_{2} C(x)$ in the nonlocal case, both existence conditions (3.15) and (4.14) can be compared by substituting $z_{0}^{2}=s+c_{2} C(x)$ into (4.14) to obtain

$$
0 \leq \frac{s+c_{2} C(x)-s}{c_{2}}(=C(x)) \leq 1+c_{1}\left(s+c_{2} C(x)\right) .
$$

This is exactly the condition (3.15) for the existence of bounded periodic solutions for the nonlocal system. This yields that bounded solutions of the nonlocal system and fast periodic solutions of the singularly perturbed system exist exactly under the same conditions.

Moreover, there is a direct relation between these two families of periodic solutions. The construction of the periodic solutions in the nonlocal case of Section 3.1 shows that these solutions are exactly the same as the uniquely defined periodic solutions of the 
unperturbed system of the singularly perturbed case which survive the perturbation. In other words, for any periodic solution of the nonlocal system there is a fast periodic solution of the singularly perturbed system which is $O(\delta)$ close. The difference can be seen most clearly by comparing $B$ in both cases: $B=$ constant in the nonlocal case, while $|B|$ varies periodically with an $O(\delta)$ amplitude around that same constant in the singularly perturbed case.

\section{Heteroclinic and Homoclinic Orbits}

In this section we will focus on heteroclinic and homoclinic orbits. From the analysis in the fast field we might expect that there exists a heteroclinic cycle for the extreme value $\frac{z_{0}^{2}-s}{c_{2}}=1+c_{1} z_{0}^{2}$, similar to the heteroclinic orbits found for the nonlocal system in Section 3.1. However, we have to be careful here: Using the Poincaré map (4.10) we can only hope to connect the one-dimensional strong unstable/stable manifolds of the critical points $\left( \pm \sqrt{\frac{c_{1} s+1}{1-c_{1} c_{2}}}, 0, \pm \sqrt{\frac{s+c_{2}}{1-c_{1} c_{2}}}, 0\right)$. Simple geometric counting arguments suggest that such connections can only exist as codimension 3 phenomena. However, using the Hamiltonian character and the symmetries (4.4) we will see in this section that these orbits always exist for $c_{2}=0$. For $c_{2} \neq 0$ there also are heteroclinic orbits; this is necessary since the periodic orbit has to disappear into some kind of heteroclinic/homoclinic structure, but these orbits will consist of fast and slow parts.

We focus on solutions of (4.3) which do consist of distinct slow and fast parts. A solution evolves slowly if it is close to a so-called slow manifold of the system. A slow manifold is an invariant manifold on which the flow is $O(\delta)$-slow. The existence of these manifolds follows from the theory originally developed by Fenichel (see [11] and references there, or [1]). There it is shown that a manifold of critical points of the unperturbed limit $\delta \rightarrow 0$, with a normally hyperbolic structure persists under the perturbation as a slow manifold. These slow manifolds play an important role in the organisation of the total flow induced by the singularly perturbed system. In this section we will construct various families of heteroclinic and homoclinic orbits which are (exponentially) close to these slow manifolds except for a number of 'jumps' through the fast field. These heteroclinic and homoclinic solutions are especially important as solutions of the full PDE (4.3) since they correspond to so-called 'localised structures' such as fronts or pulses. These localised structures are again very important for understanding the dynamics of the solutions of the PDE. We refer to [28] and the references given there for an extensive discussion of the existence and stability of these solutions in the single Ginzburg-Landau equation.

\subsection{The Slow Manifolds $\Gamma_{l}$ and $\Gamma_{r}$}

Before we apply the theory of Fenichel we note that we can find another explicit slow manifold just by setting $A=0$ in (1.5) or, equivalently, $x=y=0$ in (4.3). This is also an invariant manifold on which the flow is slow; however, its existence cannot be deduced from the general theory. By (4.6) we find that the eigenvalues of the critical point $(0,0)$ are given by $\lambda_{ \pm}= \pm i \sqrt{1+c_{1} z^{2}}$. Thus, the manifold $x=y=0$ can never 

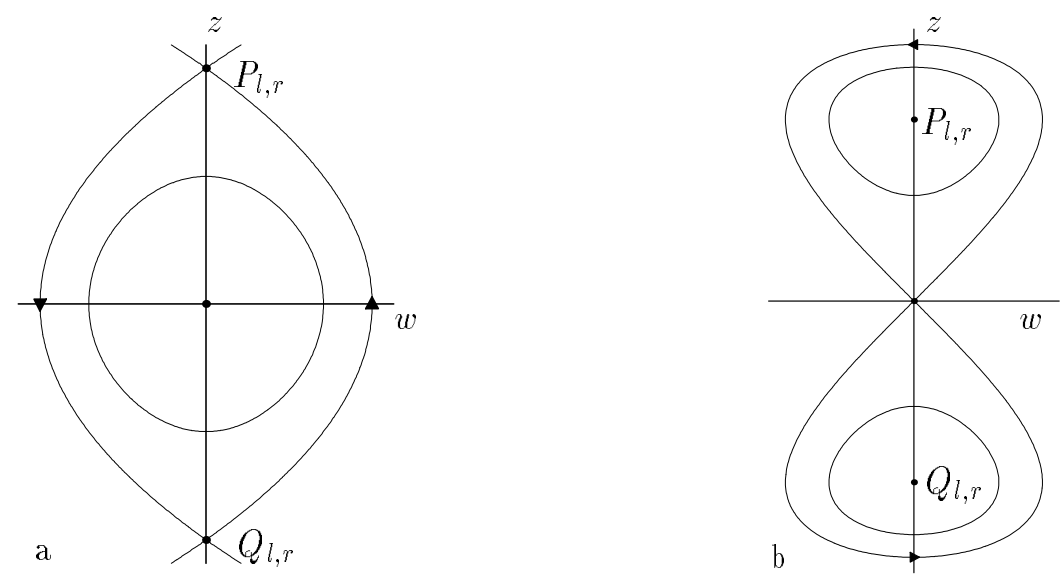

Fig. 4. The flow on the slow manifolds for (a) $s+c_{2}>0$ and (b) $s+c_{2}<0$.

be globally normally hyperbolic ( it can be normally hyperbolic for certain values of $z$ if $\left.c_{1}<0\right)$. However, the slow manifold exists since we have an explicit expression: $\{x=y=0\}$. The flow on this manifold is given by

$$
\ddot{z}=\delta^{2}\left(-s z+z^{3}\right) \text {. }
$$

Thus we note that there are two slow heteroclinic orbits between the fixed points $(0,0, \pm \sqrt{s}, 0)$ if $s>0$. There is another trivial but nonslow invariant manifold which will play a role in the forthcoming analysis: $B=0$, or $z=w=0$ in (4.3). We already encountered this manifold in the previous section.

Setting $\delta=0$ in the singularly perturbed system leads to two globally normally hyperbolic manifolds of critical points defined by $x^{2}=1+c_{1} z^{2}$ and $y=0$; see (4.6). Note that the eigenvalues of the critical points $\left( \pm \sqrt{1+c_{1} z^{2}}, 0\right)$ in (4.6) are given by $\lambda_{ \pm}= \pm \sqrt{2\left(1+c_{1} z^{2}\right)}$, and thus the invariant manifolds are globally normally hyperbolic, but only for $c_{1}>0$ are they unbounded. Thus, by the work of Fenichel [11] we know that for $\delta \neq 0$, sufficiently small, there are nearby $(=O(\delta))$ invariant slow manifolds on which the flow is $O(\delta)$. We denote these slow manifolds by $\Gamma_{l}$, for $x<0$, and by $\Gamma_{r}$, for $x>0$. The highest order approximation of the flow on the slow manifolds is given by

$$
\left\{\begin{array}{l}
\dot{z}=\delta w \\
\dot{w}=\delta\left(-\left(s+c_{2}\right) z+\left(1-c_{1} c_{2}\right) z^{3}\right),
\end{array}\right.
$$

since $x^{2}=1+c_{1} z^{2}+O(\delta)$ (see also [1]). There are several possibilities for the structure of the phase space on $\Gamma_{l}$ and $\Gamma_{r}$. The system has the following critical points: $(0,0)$ and $\left( \pm \sqrt{\frac{s+c_{2}}{1-c_{1} c_{2}}}, 0\right)$ for $\frac{s+c_{2}}{1-c_{1} c_{2}}>0$. The critical point $(0,0)$ is for $s+c_{2}>0$ a centre point and for $s+c_{2}<0$ a saddle point while for $s+c_{2}>0$ the $\left( \pm \sqrt{\frac{s+c_{2}}{1-c_{1} c_{2}}}, 0\right)$ are saddle points and for $s+c_{2}<0$ the points are centre points. One is tempted to conclude that the possible phase space is as in Figure 4. However (5.1) only gives an approximation of the flow up to $O(\delta)$ (in fact, the highest order correction turns out to be $O\left(\delta^{2}\right)$ ). On the 
other hand, we know that the system has a Hamiltonian structure; thus there are only a limited number of possibilities for the exact, integrable behaviour of the flow on the slow manifold. We use two ingredients to conclude that the topological structure of the flow on the slow manifold must be exactly as in Figure 4. First we see that the critical points on $\Gamma_{l, r}$ cannot undergo any local bifurcations (since "new" critical points on $\Gamma_{l, r}$ are also critical points of the full system and we already found all critical points). Second, we use the symmetry $\{z \rightarrow-z, w \rightarrow-w\}$ to establish the character of the heteroclinic and homoclinic connections. We focus in this section on the case $s+c_{2}>0$. However, this choice is not at all essential; we will summarise the analogous results for $s+c_{2}<0$ at the end of Section 5.3 in Remark 5.1. We denote the saddle point on $\Gamma_{l}$ (resp. $\Gamma_{r}$ ) with $z>0$ by $P_{l}$ (resp. $P_{r}$ ) and with $z<0$ by $Q_{l}$ (resp. $Q_{r}$ ),

$$
P_{l, r}, Q_{l, r}=\left( \pm \sqrt{\frac{c_{1} s+1}{1-c_{1} c_{2}}}, 0, \pm \sqrt{\frac{s+c_{2}}{1-c_{1} c_{2}}}, 0\right) .
$$

As a direct consequence of the structure of the flows on $\Gamma_{l, r}$ (Figure 4), we conclude that there exist two distinct slow heteroclinic connections between $P_{l}$ and $Q_{l}$ and between $P_{r}$ and $Q_{r}$ (for $s+c_{2}>0$ ).

The slow manifolds $\Gamma_{l}$ and $\Gamma_{r}$ possess stable and unstable manifolds, $W^{s}\left(\Gamma_{l}\right)$ and $W^{u}\left(\Gamma_{l}\right)$, resp. $W^{s}\left(\Gamma_{r}\right)$ and $W^{u}\left(\Gamma_{r}\right)$ (see again [11] and[1]). These manifolds consist of points $\left(x_{0}, y_{0}, z_{0}, w_{0}\right)$ such that the orbits $\gamma_{0}(t)$ through these points satisfy

$$
\lim _{t \rightarrow \pm \infty} \operatorname{dist}\left(\Gamma_{l, r}-\gamma_{0}(t)\right)=0
$$

where the + (resp. - ) corresponds to the stable (resp. unstable) manifold. Note that $W^{u, s}\left(\Gamma_{l, r}\right)$ merge with the manifold of stable and unstable manifolds of the (degenerate) critical points $\left( \pm \sqrt{1+c_{1} z^{2}}, 0, z, 0\right), z \in \mathbb{R}$, of the unperturbed limit (4.6). In this paper we restrict our attention to those parts of $W^{u, s}\left(\Gamma_{l, r}\right)$ which merge with the heteroclinic cycles which exist in the unperturbed limit (see Figure 5). In other words, we do not pay attention to those parts of $W^{u, s}\left(\Gamma_{l, r}\right)$ which are unbounded in the limit $\delta \downarrow 0$. Thus $W^{u}\left(\Gamma_{l}\right)=W^{s}\left(\Gamma_{r}\right)$ and $W^{u}\left(\Gamma_{r}\right)=W^{s}\left(\Gamma_{l}\right)$ in the limit $\delta \downarrow 0$. These identities fail to hold as soon as $\delta \neq 0$. However, since all $W^{u, s}\left(\Gamma_{l, r}\right)$ are three-dimensional (and the space is four-dimensional) we expect to find two-dimensional intersections $W^{u}\left(\Gamma_{l}\right) \cap W^{s}\left(\Gamma_{r}\right)$ and $W^{u}\left(\Gamma_{r}\right) \cap W^{s}\left(\Gamma_{l}\right)$.

By using the Melnikov method for slowly varying systems, the separation and thus the intersection of these stable and unstable manifolds can be calculated. See for example [27], [32]. The method is derived for the case that the unperturbed limit has homoclinic orbits, but the extension to the heteroclinic case is straightforward. Assuming that $\delta \neq 0$, sufficiently small, the distance between $W^{u}\left(\Gamma_{l}\right)$ and $W^{s}\left(\Gamma_{r}\right)$ is calculated at the cross section $\{x=0, y>0\}$. We define $y_{\delta}^{u}$ and $y_{\delta}^{s}$ as the intersection points of orbits on $W^{u}\left(\Gamma_{l}\right)$, resp. $W^{s}\left(\Gamma_{r}\right)$, with $\left\{z=z_{0}, w=w_{0}\right\}$ on $\{x=0\}$. The solutions $\gamma_{\delta}^{u}(t)=$ $\left(x_{\delta}^{u}(t), y_{\delta}^{u}(t), z_{\delta}^{u}(t), w_{\delta}^{u}(t)\right)$ in $W^{u}\left(\Gamma_{l}\right)$ and $\gamma_{\delta}^{s}(t)=\left(x_{\delta}^{s}(t), y_{\delta}^{s}(t), z_{\delta}^{s}(t), w_{\delta}^{s}(t)\right)$ in $W^{s}\left(\Gamma_{r}\right)$ of (4.3) are determined by the initial condition $\gamma_{\delta}^{u, s}(0)=\left(0, y_{\delta}^{u, s}, z_{0}, w_{0}\right) ; \gamma_{0}(t)=$ $\left(x_{0}(t), y_{0}(t), z_{0}, w_{0}\right)$ is the heteroclinic solution of the unperturbed system with $\gamma_{0}(0)=$ $\left(0, \frac{1}{2} \sqrt{2} a, z_{0}, w_{0}\right)$ where $a=1+c_{1} z_{0}^{2}$. There is an explicit expression for this solution:

$$
\left(x_{0}(t), y_{0}(t)\right)=\left(\sqrt{2} b \tanh (b t), \sqrt{2} b^{2}\left(1-\tanh ^{2}(b t)\right)\right),
$$



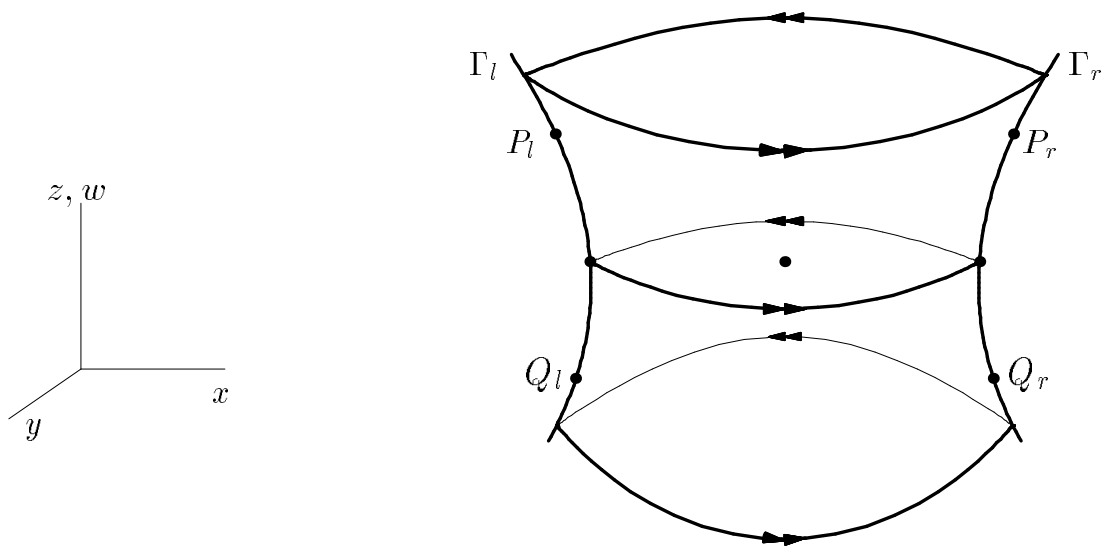

Fig. 5. A three-dimensional sketch of the four-dimensional phase space of the unperturbed system. The two-dimensional slow manifolds $\Gamma_{l}$ and $\Gamma_{r}$ are represented by curves. The positions of the critical points of the perturbed system are also indicated.

where $b=\frac{1}{2} \sqrt{2\left(1+c_{1} z_{0}^{2}\right)}$. As usual in Melnikov theory, we define the following timedependent distance function:

$$
\Delta\left(t, z_{0}, w_{0}\right)=\left(\begin{array}{c}
\frac{\partial}{\partial \delta}\left(x_{\delta}^{u}(t)-x_{\delta}^{s}(t)\right) \\
\frac{\partial}{\partial \delta}\left(y_{\delta}^{u}(t)-y_{\delta}^{s}(t)\right)
\end{array}\right) \wedge\left(\begin{array}{c}
y_{0}(t) \\
-x_{0}(t)+x_{0}^{3}(t)-c_{1} x_{0}(t) z_{0}^{2}
\end{array}\right),
$$

where the wedge product represents the scalar cross-product in the plane. The distance between $W^{u}\left(\Gamma_{l}\right)$ and $W^{s}\left(\Gamma_{r}\right)$ in the $\left\{z=z_{0}, w=w_{0}\right\}$-plane is given by $\Delta\left(0, z_{0}, w_{0}\right)$. Similar to the derivation of the Melnikov function for periodically driven systems, one shows (see [27]) that for (4.3),

$$
\begin{aligned}
\Delta\left(0, z_{0}, w_{0}\right) & \stackrel{\text { def }}{=} \Delta\left(z_{0}, w_{0}\right) \\
& =\int_{-\infty}^{\infty}\left(\begin{array}{c}
0 \\
-2 c_{1} x_{0}(t) z_{0} \frac{\partial z}{\partial \delta}(t)
\end{array}\right) \wedge\left(\begin{array}{c}
y_{0}(t) \\
-x_{0}(t)+x_{0}^{3}(t)-c_{1} x_{0}(t) z_{0}^{2}
\end{array}\right) d t,
\end{aligned}
$$

where $\frac{\partial z}{\partial \delta}(t)$ is a solution of $\frac{d}{d t} \frac{\partial z}{\partial \delta}=w_{0}$ with $\frac{\partial z}{\partial \delta}(0)=0$; thus $\frac{\partial z}{\partial \delta}=w_{0} t$. The separation is then given by

$$
\Delta\left(z_{0}, w_{0}\right)=-2 c_{1} w_{0} z_{0} \int_{-\infty}^{\infty} t x_{0}(t) y_{0}(t) d t
$$

where $\left(x_{0}(t), y_{0}(t)\right)$ is the heteroclinic solution of the unperturbed system. Substituting this expression into (5.4) results in

$$
\Delta\left(z_{0}, w_{0}\right)=-4 c_{1} w_{0} z_{0} b^{3} \int_{-\infty}^{\infty} t \tanh (b t)\left(1-\tanh ^{2}(b t)\right) d t .
$$

Since

$$
\int_{-\infty}^{\infty} t \tanh (b t)\left(1-\tanh ^{2}(b t)\right) d t=\frac{1}{b^{2}}
$$




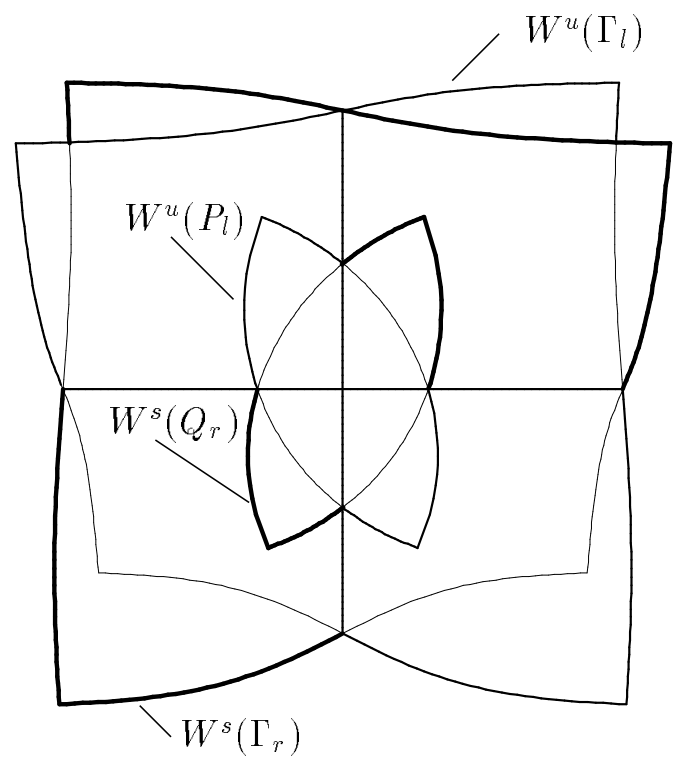

Fig. 6. The intersection of the two-dimensional manifolds $W^{u}\left(\Gamma_{l}\right) \cap\{x=0, y>0\}$ and $W^{s}\left(\Gamma_{r}\right) \cap\{x=$ $0, y>0\}$ for $c_{1}<0$ and $c_{2}<0$. Parts of the onedimensional curves $W^{u}\left(P_{l}, Q_{l}\right) \cap\{x=0, y>0\} \subset$ $W^{u}\left(\Gamma_{l}\right) \cap\{x=0, y>0\}$ and $W^{s}\left(P_{r}, Q_{r}\right) \cap\{x=$ $0, y>0\} \subset W^{s}\left(\Gamma_{r}\right) \cap\{x=0, y>0\}$ are also shown.

we obtain (in leading order)

$$
\Delta\left(w_{0}, z_{0}\right)=-2 c_{1} w_{0} z_{0} \sqrt{2\left(1+c_{1} z_{0}^{2}\right)} .
$$

This yields that $W^{u}\left(\Gamma_{l}\right) \cap W^{s}\left(\Gamma_{r}\right) \cap\{x=0, y>0\}$ is $O(\delta)$ close to either the $\left\{w_{0}=0\right\}$ plane or the $\left\{z_{0}=0\right\}$-plane. See Figure 6 for a sketch of this intersection. Using the symmetries (4.4) one derives a similar expression for $W^{s}\left(\Gamma_{l}\right) \cap W^{u}\left(\Gamma_{r}\right) \cap x=0, y<0$.

So far, we have found a number of trivial heteroclinic connections, such as those found in the invariant planes $\{x=y=0\}$ and $\{z=w=0\}$. Note that the former pair between the points $(-1,0,0,0) \in \Gamma_{l}$ and $(1,0,0,0) \in \Gamma_{r}$ corresponds to the zeros of $(5.6)$ at $z_{0}=$ $w_{0}=0$ for $W^{u}\left(\Gamma_{l}\right) \cap W^{s}\left(\Gamma_{r}\right) \cap\{x=0\}$ and $W^{s}\left(\Gamma_{l}\right) \cap W^{u}\left(\Gamma_{r}\right) \cap\{x=0\}$. Two additional pairs of heteroclinic orbits are found in $\Gamma_{l}$ and $\Gamma_{r}$. These orbits connect $P_{l}$ to $Q_{l}$, resp. $P_{r}$ to $Q_{r}$; we denote these orbits as $\gamma_{P_{l} Q_{l}}^{(0)}(t), \gamma_{Q_{l} P_{l}}^{(0)}(t) \in \Gamma_{l}$, where $\lim _{t \rightarrow \infty} \gamma_{P_{l} Q_{l}}^{(0)}(t)$ (resp. $\left.\gamma_{Q_{l} P_{l}}^{(0)}(t)\right)=Q_{l}$ (resp. $\left.P_{l}\right)$, and analogously $\gamma_{P_{r} Q_{r}}^{(0)}(t), \gamma_{Q_{r} P_{r}}^{(0)}(t) \in \Gamma_{r}$. The flow on these orbits is, of course, everywhere $O(\delta)$.

\subsection{The Fundamental Heteroclinic Orbits}

Next, we will construct heteroclinic orbits between the points $P_{l, r}, Q_{l, r}$ which consist of distinct slow and fast parts. Based on these orbits we will construct multijump orbits. We will do that by analysing the intersections of the stable and unstable manifolds of 
these points with the $\{x=0\}$-plane. These manifolds are subsets of $W^{s, u}\left(\Gamma_{l}, \Gamma_{r}\right)$. The 'toffee'-like structure in $\Gamma_{l}$ formed by the unstable manifolds of $P_{l}$ and $Q_{l}$ will be mapped by the flow on $W^{u}\left(P_{l}\right) \cup W^{u}\left(Q_{l}\right)$ to a topologically similar structure which is contained in $W^{u}\left(\Gamma_{l}\right) \cap\{x=0\}$ (moreover, the $z$-and $w$-coordinates are only $O(\delta)$ modified by the fast field). Thus, by (5.6), there will be four intersection points with $W^{s}\left(\Gamma_{r}\right) \cap\{x=0\}$ (two near $z_{0}=0$ and two near $w_{0}=0$ ). Note that all four intersections are transversal. These intersection points correspond to orbits which are biasymptotic to $\Gamma_{l}$ and $\Gamma_{r}$. By construction, they satisfy $\lim _{t \rightarrow-\infty}=P_{l}$ or $Q_{l}$. These orbits are all on the same energy level set as $P_{l}$ and $Q_{l}$. Thus, by the Hamiltonian structure of the flow, they can only be asymptotic to orbits on $\Gamma_{r}$ with that same energy: the stable and unstable manifolds of $P_{r}$ and $Q_{r}$. This indicates that the four orbits are heteroclinic connections between $P_{l}, Q_{l}$ and $P_{r}, Q_{r}$. A similar argument yields four connections which travel from $\Gamma_{r}$ to $\Gamma_{l}$. However, we have to use the symmetries (4.4) of the system to get a more precise result:

Theorem 1. For any $s, c_{1}$, and $c_{2}$ which satisfy

$$
1+c_{1} s>0, \quad 1-c_{1} c_{2}>0, \quad \text { and } s+c_{2}>0,
$$

eight heteroclinic orbits of the following type exist (in (4.3)):

$$
\begin{array}{rcc}
\gamma_{L R}^{(1)}(t) \text { with } & \lim _{t \rightarrow-\infty} \gamma_{L R}^{(1)}(t)=L, & \lim _{t \rightarrow \infty} \gamma_{L R}^{(1)}(t)=R \\
& \text { and } L=P_{l}, Q_{l} ; & R=P_{r}, Q_{r} . \\
\gamma_{R L}^{(1)}(t) \text { with } & \lim _{t \rightarrow-\infty} \gamma_{R L}^{(1)}(t)=R, & \lim _{t \rightarrow \infty} \gamma_{R L}^{(1)}(t)=L \\
& \text { and } L=P_{l}, Q_{l} ; & R=P_{r}, Q_{r} .
\end{array}
$$

All eight orbits consist of three parts: two slow parts near either $\Gamma_{l}$ or $\Gamma_{r}$ and one 'jump' through the fast field.

Note that condition (5.7) just ascertains the existence of the critical points $P_{l, r}, Q_{l, r}$ in the case $s+c_{2}>0$. Schematic sketches of all eight orbits are given in Figures 7, 8, and 9.

Proof. First we prove the existence of $\gamma_{P_{l} Q_{r}}^{(1)}(t)$. The existence of the three orbits $\gamma_{P_{r} Q_{l}}^{(1)}(t)$, $\gamma_{Q_{l} P_{r}}^{(1)}(t)$, and $\gamma_{Q_{r} P_{l}}^{(1)}(t)$ follows from the symmetries $\{x \rightarrow-x, y \rightarrow-y\},\{z \rightarrow$ $-z, w \rightarrow-w\}$, and $\{t \rightarrow-t, y \rightarrow-y, w \rightarrow-w\}$. Second, we turn our attention to proving the existence of $\gamma_{P_{l} P_{r}}^{(1)}(t)$. The remaining three orbits follow from this one by using the symmetries.

Let $\gamma_{p}(t)=\left(x_{p}(t), y_{p}(t), z_{p}(t), w_{p}(t)\right)$ be a solution of (4.3) on $W^{u}\left(P_{l}\right)$ which is exponentially close to $\Gamma_{l}$ between the points $P_{l}$ and $p=\left(p_{x}, p_{y}, p_{z}, p_{w}\right) \in W^{u}\left(P_{l}\right) \cap$ $W^{s}\left(Q_{l}\right) \cap \Gamma_{l}$ (thus, $\gamma_{p}$ leaves $\Gamma_{l} O(\delta)$ near $\left.p\right)$. Note that $W^{u}\left(P_{l}\right) \cap W^{s}\left(Q_{l}\right) \cap \Gamma_{l}$ is the above-defined 'trivial' heteroclinic orbit $\gamma_{P_{l} Q_{l}}^{(0)}$. We denote by $\gamma_{p}(0)$ the (first) intersection of $\gamma_{p}$ with the $\{x=0\}$-plane: $\gamma_{p}(0)=\left(0, y_{p}(0), z_{p}(0), w_{p}(0)\right)$. Using the symmetries (4.4) we define the orbits $\gamma_{p}^{z}(t)=\left(-x_{p}(-t), y_{p}(-t),-z_{p}(-t), w_{p}(-t)\right)$ and $\gamma_{p}^{w}(t)=$ $\left(-x_{p}(-t), y_{p}(-t), z_{p}(-t),-w_{p}(-t)\right)$. Note that $\lim _{t \rightarrow \infty} \gamma_{p}^{z}(t)=Q_{r}, \lim _{t \rightarrow \infty} \gamma_{p}^{w}(t)=$ $P_{r}$, and that $\gamma_{p}^{z}(0)=\left(0, y_{p}(0),-z_{p}(0), w_{p}(0)\right), \gamma_{p}^{w}(0)=\left(0, y_{p}(0), z_{p}(0),-w_{p}(0)\right)$. 


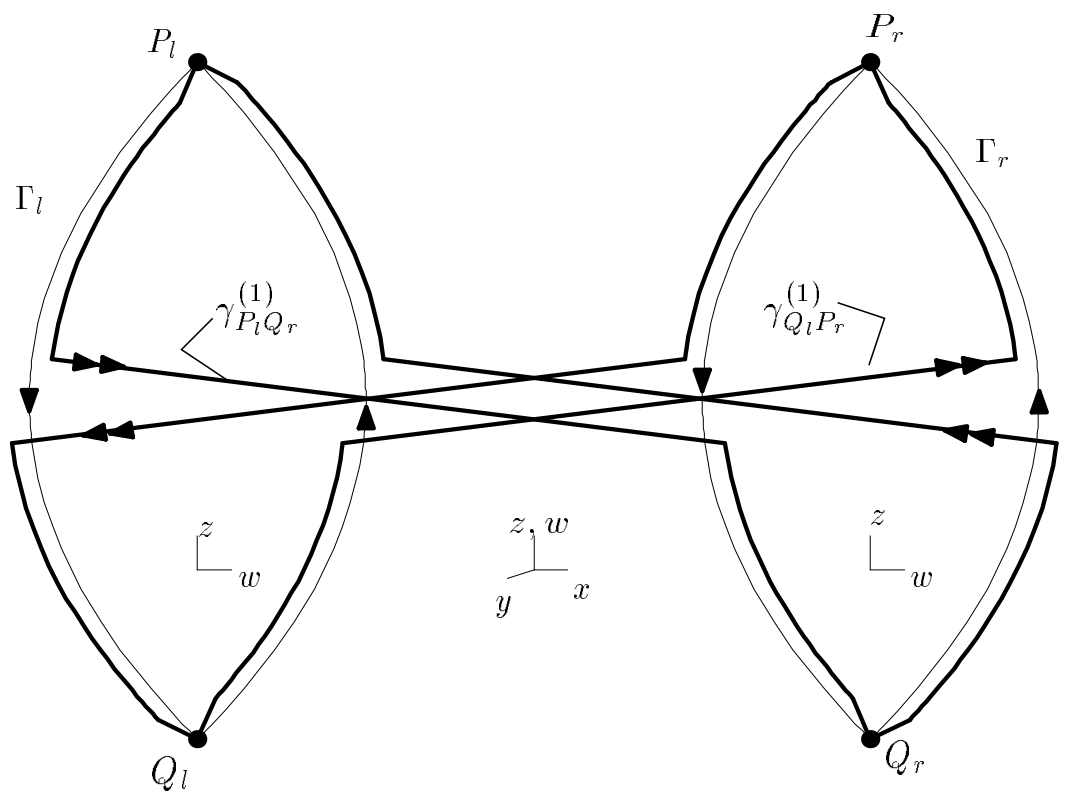

Fig. 7. A schematic sketch of the four one-jump orbits $\gamma_{P_{l} Q_{r}}^{(1)}, \gamma_{P_{r} Q_{l}}^{(1)}, \gamma_{Q_{l} P_{r}}^{(1)}$, and $\gamma_{Q_{r} P_{s}}^{(1)}$. The slow parts are exponentially close to the heteroclinic cycles on $\Gamma_{l, r}$ (the thin lines). The fast parts 'jump' through the full four-dimensional phase-space, $O(\delta)$ close to the $\{z=0\}$-hyperplane. These orbits exist for every $c_{2}$.

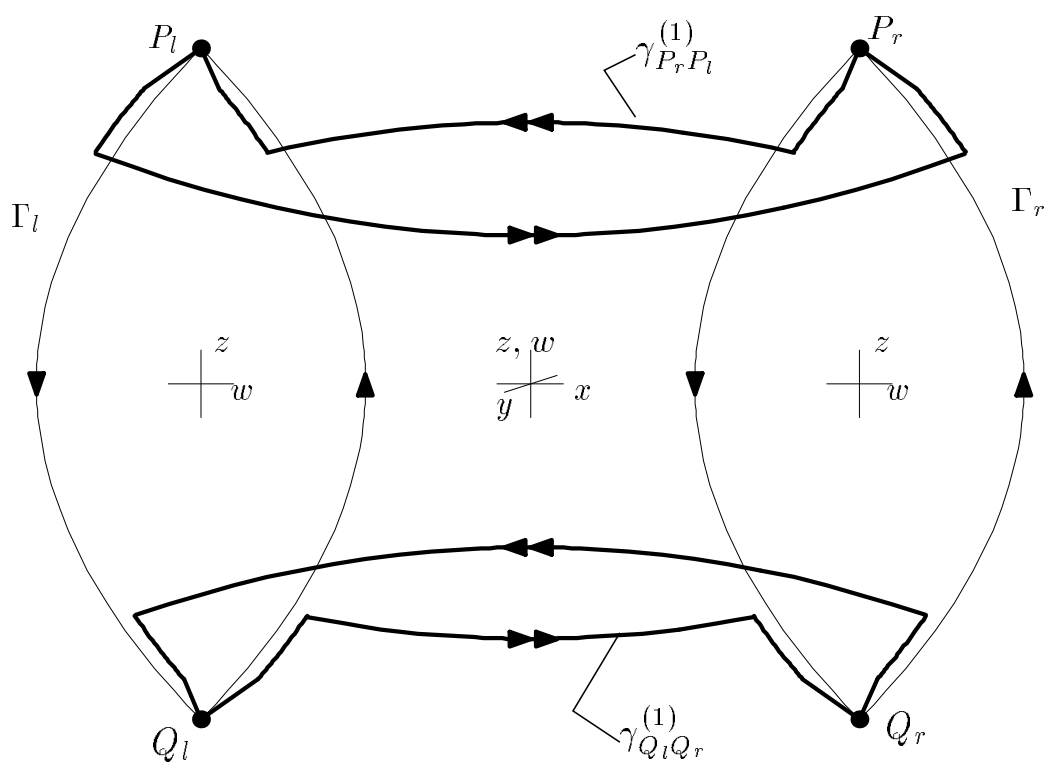

Fig. 8. A schematic sketch of the four one-jump orbits $\gamma_{P_{l} P_{r}}^{(1)}(t), \gamma_{P_{r} P_{l}}^{(1)}(t), \gamma_{Q_{l} Q_{r}}^{(1)}(t)$, and $\gamma_{Q_{r} Q_{l}}^{(1)}(t)$ for $c_{2}>0$ which 'jump' $O(\delta)$ close to the $\{w=0\}$-hyperplane. 


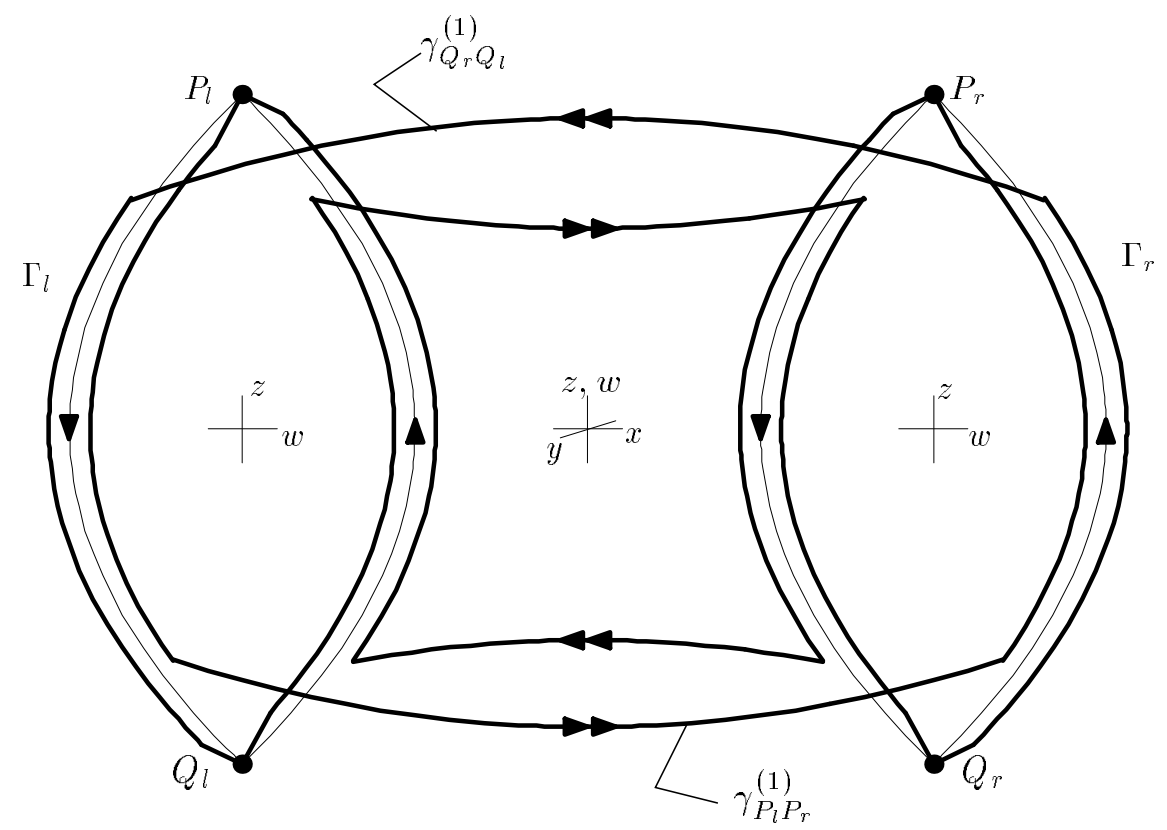

Fig. 9. A schematic sketch of the four orbits $\gamma_{P_{l} P_{r}}^{(1)}(t), \gamma_{P_{r} P_{l}}^{(1)}(t), \gamma_{Q_{l} Q_{r}}^{(1)}(t)$, and $\gamma_{Q_{r} Q_{l}}^{(1)}(t)$ for $c_{2}<0$ which 'jump' $O(\delta)$ close to the $\{w=0\}$-hyperplane.

Thus, the symmetries of (4.3) yield a heteroclinic solution between $P_{l}$ and $Q_{r}$ (resp. $P_{r}$ ) if we can choose $p$ such that $z_{p}(0)=0\left(\right.$ resp. $\left.w_{p}(0)=0\right)$, since then $\gamma_{p}^{z}\left(\right.$ resp. $\left.\gamma_{p}^{w}\right)$ coincides with $\gamma_{p}$ at $t=0$.

The fast field between $p$ and $\left(0, y_{p}(0), z_{p}(0), w_{p}(0)\right)$ only has an $O(\delta)$ influence on the slow $(z, w)$-coordinates of $\gamma_{p}: z_{p}(0)$ is $O(\delta)$ close to the $z$-coordinate $p_{z}$ of $p \in \Gamma_{l}$. Since $p_{z}$ can be varied between the $z$-coordinates of $P_{l}$ and $Q_{l}(5.2)$, we see that there must be a $p_{z}^{*}$ such that $\gamma_{p^{*}}=\gamma_{p^{*}}^{z}$ is a heteroclinic solution between $P_{l}$ and $Q_{r}$ of the type described by the Theorem. By the symmetries $\{x \rightarrow-x, y \rightarrow-y\},\{z \rightarrow-z, w \rightarrow-w\}$, and $\{t \rightarrow-t, y \rightarrow-y, w \rightarrow-w\}$, three distinct, symmetric counterparts of $\gamma_{p^{*}}$ can be constructed. Thus, we have proved the existence of the solutions $\gamma_{P_{l} Q_{r}}^{(1)}, \gamma_{P_{r} Q_{l}}^{(1)}, \gamma_{Q_{l} P_{r}}^{(1)}$, and $\gamma_{Q_{r} P_{l}}^{(1)}$ described in the Theorem (see Figure 7).

One has to be more careful in constructing the other four orbits. First we have to construct a connection between $P_{l}$ and $P_{r}$ by determining a $p$ such that $\gamma_{p}(t)$ and $\gamma_{p}^{w}(t)$ can be identified. Thus, we have to find a $p \in W^{u}\left(P_{l}\right) \cap W^{s}\left(Q_{l}\right) \cap \Gamma_{l}$ such that $w_{p}(0)=0$. However, the $w$-coordinate of $p, w_{p}$ does not change sign. Since the $w$-coordinates of $P_{l}$ and $Q_{l}$ are 0 and $w_{p}(0)$ is $O(\delta)$ close to $w_{p}$, we can only expect to find a heteroclinic connection between $P_{l}$ and $P_{r}$ if we choose $p O(\delta)$ close to $P_{l}$ or $Q_{l}$. It is possible to compute $w_{p}(0)$ up to $O\left(\delta^{2}\right)$ accuracy for these values of $p$ by the Poincaré map $P$ (4.10). We set $k=0$ and $(z, w)=$ the coordinates of $P_{l}$ or $Q_{l}$ (5.2) in (4.1), where we have to change the interval of integration $\left(-T_{\delta}, T_{\delta}\right)$ into $(-\infty, 0)$. This way $P$ measures the accumulated change in $k, z, w$ on the one-dimensional (purely) strong unstable manifolds of $P_{l}$ and $Q_{l}$ between $\Gamma_{l}$ and $\{x=0\}$. Note that all three integrals converge and that $\Delta K$ 
and $\Delta K_{1}$ are $0+O\left(\delta^{2}\right) ;$ the computation of $\Delta K_{2}$ yields

$$
w_{p}(0)= \pm \sqrt{2} \delta c_{2} \sqrt{\frac{1+c_{1} s}{1-c_{1} c_{2}}} \sqrt{\frac{s+c_{2}}{1+s c_{1}}},
$$

where the $+\left(\right.$ resp. - ) sign corresponds to $p=P_{l}$ (resp. $Q_{l}$ ). Note that $c_{2}$ is the only parameter which has influence on the sign of $w_{p}(0)$. However, by (5.8), we observe that for all $c_{2} \neq 0, w_{p}(0)$ has to change sign (at least) once if $p$ is varied from $P_{l}$ to $Q_{l}$. Moreover, if $c_{2}=0$ we see that $(\dot{z}, \dot{w})$ decouple from the $(\dot{x}, \dot{y})$ in $(4.3)$ : There are exact (integrable) connections between the one-dimensional strong stable/unstable manifolds of $P_{l}$ and $P_{r}$ if $z \equiv$ the $z$ - coordinate of $P_{l}$ in (5.2). Thus there exists a $p \in W^{u}\left(P_{l}\right) \cap W^{s}\left(Q_{l}\right) \cap \Gamma_{l}$ such that $w_{p}(0)=0$, and for this $p: \gamma_{p}=\gamma_{p}^{w}$. We conclude that there exists a heteroclinic orbit between $P_{l}$ and $P_{r}$ for all $c_{2}$ such that (5.7) holds. As in the previous case, we can construct from this orbit, $\gamma_{P_{l} P_{r}}^{(1)}(t)$, three symmetrical counterparts, $\gamma_{P_{r} P_{l}}^{(1)}(t), \gamma_{Q_{l} Q_{r}}^{(1)}(t)$, and $\gamma_{Q_{r} Q_{l}}^{(1)}(t)$ by applying the symmetries $\{x \rightarrow-x, y \rightarrow-y\},\{z \rightarrow-z, w \rightarrow-w\}$, and $\{t \rightarrow-t, y \rightarrow-y, z \rightarrow-z\}$. This concludes the proof of the Theorem (see Figures 8, 9).

At this point we can compare the behaviour of the 'localised structures' in the nonlocal and in the singularly perturbed system. We ignore, just for the moment, the fact that we derived the extra condition (1.7). Remember that the heteroclinic orbits found in Section 3.1 do not satisfy the extra condition (1.7). First, we note that the solutions $\gamma_{P_{l} Q_{r}}^{(1)}$, $\gamma_{P_{r} Q_{l}}^{(1)}, \gamma_{Q_{l} P_{r}}^{(1)}$, and $\gamma_{Q_{r} P_{l}}^{(1)}$, which intersect $\{x=0\}$ at $z=0$, cannot have a counterpart in the nonlocal system, simply because the $w$-coordinates of these solutions are $O(1)$ during the fast 'jump' while all solutions of the nonlocal system must correspond to $w=\dot{z}=0$. The other four solutions have a $w$-coordinate of $O(\delta)$ during the jump. Moreover, the jumps take place $O(\delta)$ near $P_{l}$ and $P_{r}$ (or $Q_{l}$ and $Q_{r}$ ) and it is easy to check that the jumps are $O(\delta)$ close to the two pairs of nonlocal, integrable heteroclinic orbits found in Section 3.1. Thus, it is natural to conclude that $\gamma_{P_{l} P_{r}}^{(1)}(t), \gamma_{P_{r} P_{l}}^{(1)}(t), \gamma_{Q_{l} Q_{r}}^{(1)}(t)$, and $\gamma_{Q_{r} Q_{l}}^{(1)}(t)$ are the counterparts of the nonlocal heteroclinic orbits.

However, this conclusion can only be justified if the coupling coefficient, $c_{2}$, in the $B$-equation, is positive. This follows from (5.8): The jump of the connection $\gamma_{p}(t)$ takes place $O(\delta)$ near $P_{l}$ if $c_{2}>0$. Thus all four heteroclinic orbits are as in Figure 8: They are $O(\delta)$ close to the purely fast connections of the unperturbed or the nonlocal problem. The parts of the orbits near $\Gamma_{l}$ and $\Gamma_{r}$ are only of an $O(\delta)$ length. If $c_{2}<0$ the connection $\gamma_{P_{l} P_{r}}^{(1)}(t)$ makes its jump $O(\delta)$ near the unperturbed, fast connections between $Q_{l}$ and $Q_{r}$. The solution follows $W^{u}\left(P_{l}\right) \cap W^{s}\left(Q_{l}\right) \cap \Gamma_{l}$ from $P_{l}$ almost $(O(\delta))$ up to $Q_{l}$. The same happens in/near $\Gamma_{r}$ (see Figure 9). Analogously, the unperturbed fast connections between $P_{l}$ and $P_{r}$ correspond to heteroclinic orbits from $Q_{l}$ to $Q_{r}$ of the perturbed system. Moreover, the $w$-coordinate of these solutions becomes $O(1)$ during their (long) stays near $\Gamma_{l}$ and $\Gamma_{r}$. Note that this significant distinction between the cases $c_{2}>0$, $c_{2}<0$ also has its impact on the periodic orbits found in Section 3.1: These solutions will have to merge with the heteroclinic cycles $\left\{\gamma_{P_{l} P_{r}}^{(1)}(t), \gamma_{P_{r} P_{l}}^{(1)}(t)\right\}$ and $\left\{\gamma_{Q_{l} Q_{r}}^{(1)}(t), \gamma_{Q_{r} Q_{l}}^{(1)}(t)\right\}$ as $z_{0}$ approaches $\pm \sqrt{\left(c_{2}+s\right) /\left(1-c_{1} c_{2}\right)}$, the $z$-coordinates of $P_{l, r}, Q_{l, r}$ (see (5.2)). For $c_{2}<0$ the periodic orbits with $z_{0}>0$ (resp. $z_{0}<0$ ) will 'grow' large, slow parts (exponentially) close to $\Gamma_{l}$ and $\Gamma_{r}$ which follow the cycle $\left\{\gamma_{Q_{l} Q_{r}}^{(1)}(t), \gamma_{Q_{r} Q_{l}}^{(1)}(t)\right\}$ (resp. 
$\left.\left\{\gamma_{P_{l} P_{r}}^{(1)}(t), \gamma_{P_{r} P_{l}}^{(1)}(t)\right\}\right)$ as $z_{0}$ approaches $\sqrt{\left(c_{2}+s\right) /\left(1-c_{1} c_{2}\right)}$ (see Figure 9). In a sense one can interpret this as a four-dimensional 'canard-like' behaviour (see [8]), since this $O(1)$ change in the periodic orbits takes place for an exponentially small change in $z_{0}$. Thus, a significant part of the structure of the solutions disappears in the transition from the nonlocal system to the singularly perturbed system if $c_{2}<0$.

\subsection{Multijump Heteroclinic Orbits}

In this subsection we will study the possible existence of 'multijump' heteroclinic orbits, that is, heteroclinic solutions connecting two of the critical points $P_{l, r}, Q_{l, r}$ by various jumps through the fast field alternated with slow parts near $\Gamma_{l}, \Gamma_{r}$. These solutions cannot have a counterpart in the nonlocal limit. We will find that they can only exist for $c_{2}<0$. First we focus on orbits which only make jumps from $\Gamma_{l}$ to $\Gamma_{r}$, or vice versa, without following the periodic flow of the fast field for more than half a circuit. Later, we will construct orbits from $\Gamma_{l}$ (or $\Gamma_{r}$ ) to itself which make one complete circuit through the fast field.

Before we formulate the Theorem on the existence of multijump orbits of the first type we give a construction of one of the most simple multi-=2-jump heteroclinic orbits in the case $c_{2}<0$ and show that this construction cannot work if $c_{2}>0$.

We consider the part of the two-dimensional unstable manifold $W^{u}\left(P_{l}\right)$ on which the orbits approach $P_{l}$ (as $t \rightarrow-\infty$ ) 'from the right' tangential to the trivial heteroclinic orbit $\gamma_{P_{l} Q_{l}}^{(0)}$. Thus, as in the definition of $W^{u}\left(\Gamma_{l}\right)$, we only consider those parts of $W^{s, u}\left(P_{l, r} Q_{l, r}\right)$ which merge with the family of heteroclinic connections in the limit $\delta \downarrow 0$. For simplicity we also denote this subset of $W^{u}\left(\Gamma_{l}\right)$ by $W^{u}\left(P_{l}\right)$. In the sequel we will use similar restrictions on the 'full' manifolds $W^{u, s}\left(P_{l, r}, Q_{l, r}\right)$, also without adapting the notation. By the above Theorem we know that $W^{u}\left(P_{l}\right)$ intersects $W^{s}\left(P_{r}\right)$ : This is the orbit $\gamma_{P_{l} P_{r}}^{(1)}(t)$ which has, if $c_{2}<0$ (resp. $\left.c_{2}>0\right),(z, w)$-coordinates $O(\delta)$ close to those of $Q_{l}$ (resp. $P_{l}$ ) during its jump through the fast field.

First we consider the case $c_{2}<0$. Let $\mathcal{L}_{1} \subset W^{u}\left(P_{l}\right) \cap\{x=0\}$ be a (one-dimensional) neighbourhood of $\gamma_{P_{l} P_{r}}^{(1)} \cap\{x=0\} ; \mathcal{L}_{1}$ intersects $W^{s}\left(P_{r}\right)$ transversally (by (5.6)). Define for $q \in \mathcal{L}_{1}$ the orbit through $q$ by $\gamma_{q}(t) \in W^{u}\left(P_{l}\right)$. Thus when $q_{0}=\mathcal{L}_{1} \cap W^{s}\left(P_{r}\right), \gamma_{q_{0}}=$ $\gamma_{P_{l} P_{r}}^{(1)}$. The orbit $\gamma_{q}$ will follow $\gamma_{q_{0}}$ along $\Gamma_{r}$ for an $O(1)$ distance, if $q$ is exponentially close to $q_{0}$. Such an orbit $\gamma_{q}$ will leave the neighbourhood of $\Gamma_{r}$ exponentially close to $W^{u}\left(Q_{r}\right)$ since $\gamma_{q_{0}} \in W^{s}\left(P_{r}\right)$ and $W^{u}\left(Q_{r}\right) \cap \Gamma_{r}=W^{s}\left(P_{r}\right) \cap \Gamma_{r}$ (see Figure 9). We take $\mathcal{L}_{1}$ of exponentially short length; $\mathcal{L}_{1}$ is divided into two distinct parts by $W^{s}\left(\Gamma_{r}\right) \cap\{x=0\}$ with $q_{0}=\gamma_{P_{l} P_{r}}^{(1)} \cap\{x=0\}$ as separatrix. Therefore, the two-dimensional manifold $\mathcal{F}\left(\mathcal{L}_{1}\right)$ of orbits $\gamma_{q}$ through $\mathcal{L}_{1}$ is separated into two parts, an 'inner' and an 'outer' part, by the three-dimensional stable manifold $W^{s}\left(\Gamma_{r}\right)$ of $\Gamma_{r}$ before it approaches $\Gamma_{r}$. Orbits $\gamma_{q}(t)$ on the outer part of $\mathcal{F}\left(\mathcal{L}_{1}\right)$ will again leave the neighbourhood of $\Gamma_{r}$ in the direction opposite to $\Gamma_{l}$ (their $x$-coordinates increase): They cannot return to either $\Gamma_{l}$ or $\Gamma_{r}$ and become unbounded. Orbits on the inner part of $\mathcal{F}\left(\mathcal{L}_{1}\right)$ will follow $W^{u}\left(\Gamma_{r}\right)$-where we use the restricted definition (see above) - and return to the $\{x=0\}$-hyperplane. The flow near $\Gamma_{r}$ twists $\mathcal{F}\left(\mathcal{L}_{1}\right)$ such that the inner part leaves the neighbourhood of $\Gamma_{r}$ as a 'sheet' exponentially close to $W^{u}\left(Q_{r}\right)$. We refer to [14] and especially [15] (since this paper applies to system (1.5) for a general treatment of the deformation of manifolds 
near slow manifolds; see also [1]). Thus, the intersection $\mathcal{F}\left(\mathcal{L}_{1}\right) \cap\{x=0\}$, after the (first) passage through a neighbourhood of $\Gamma_{r}$, consists of a curve exponentially close to the curve $W^{u}\left(Q_{r}\right) \cap\{x=0\} \subset W^{u}\left(\Gamma_{r}\right) \cap\{x=0\}$. By Theorem 1 we know that $W^{u}\left(Q_{r}\right) \cap\{x=0\}$ intersects the two-dimensional manifold $W^{s}\left(\Gamma_{l}\right) \cap\{x=0\}$ twice transversally (the orbit $\gamma_{Q_{r} P_{l}}^{(1)}$ near $\{z=0\}$ [Figure 7] and the orbit $\gamma_{Q_{r} Q_{l}}^{(1)}$ near $\{w=0\}$ [Figure 9]). Now we note that the orbit $\gamma_{q_{0}}=\gamma_{P_{l} P_{r}}^{(1)}$ already 'touches down' on $\Gamma_{r}$ (or better: approaches $\Gamma_{r}$ exponentially close) $O(\delta)$ close to $Q_{r}$. Thus, $\gamma_{q_{0}}$ is exponentially close to $\gamma_{Q_{r} P_{l}}^{(1)}$ and $\gamma_{Q_{r} Q_{l}}^{(1)}$ for certain parts of $O(1)$ length of these orbits (near $\Gamma_{r}$ ). As a consequence, we know that the curve $\mathcal{F}\left(\mathcal{L}_{1}\right) \cap\{x=0\}$ must be extended along the entire length of $W^{u}\left(Q_{r}\right) \cap\{x=0\}$ (except for an $O(\delta)$ part), and that it thus has to intersect $W^{s}\left(\Gamma_{l}\right) \cap\{x=0\}$ twice, exponentially close to $\gamma_{Q_{r} P_{l}}^{(1)}$ and $\gamma_{Q_{r} Q_{l}}^{(1)}$. This yields that there are two orbits on $\mathcal{F}\left(\mathcal{L}_{1}\right)$ which are asymptotic to $\Gamma_{l}$. We again apply the argument that system (4.3) has a Hamiltonian structure, so that the 'energy' $H$ is conserved on orbits: $\mathcal{F}\left(\mathcal{L}_{1}\right)$ can only intersect $W^{s}\left(\Gamma_{l}\right)$ along $W^{s}\left(P_{l}\right)$ or $W^{s}\left(Q_{l}\right)$.

So we conclude that there exist two two-jump orbits, which consist of five parts: a slow part near $\Gamma_{l}$, a fast jump, a slow part near $\Gamma_{r}$, a second jump, and a third slow part near $\Gamma_{l}$ : the heteroclinic orbit $\gamma_{P_{l} Q_{l}}^{(2,1)}$ and the homoclinic orbit $\gamma_{P_{l} P_{l}}^{(2,1)}$. By the symmetries (4.4) we can create a family of eight distinct two-jump homoclinic orbits and four twojump heteroclinic orbits. Note that there thus exist, for instance, two different homoclinic two-jump orbits to $P_{l}, \gamma_{P_{l} P_{l}}^{(2,1)}$ and $\gamma_{P_{l} P_{l}}^{(2,2)}$, related to each other by the symmetry $\{y \rightarrow$ $-y, t \rightarrow-t, w \rightarrow-w\}$ (see below).

Before we extend the above argument to $3,4, \ldots$-jump heteroclinic and homoclinic orbits we consider the case $c_{2}>0$. The above construction is impossible in this case. The construction is based on the orbit $\gamma_{P_{l} P_{r}}^{(1)}$. This orbit exists also for $c_{2}>0$, but now, as we already noted above, this orbit only has parts of $O(\delta)$ length near the slow manifolds (Figure 8). Thus, the intersection of $\mathcal{F}\left(\mathcal{L}_{1}\right)$ with $\{x=0\}$ (after passing $\Gamma_{r}$ ) is also only of $O(\delta)$ length and cannot intersect $W^{s}\left(\Gamma_{l}\right) \cap\{x=0\}: \mathcal{F}\left(\mathcal{L}_{1}\right) \cap W^{s}\left(\Gamma_{l}\right)=\emptyset$. Of course one could try to construct two-jump orbits based on one of the one-jump orbits which jump near $\{z=0\}$ after following a trivial heteroclinic orbit on $\Gamma_{l}$ for half its length (Figure 7). Let's for instance consider $\gamma_{P_{l} Q_{r}}^{(1)}$ (this is no restriction, due to the symmetries (4.4)). It is only possible to construct a two-jump orbit if $\gamma_{P_{l} Q_{r}}^{(1)}$ has parts exponentially close to one of the two one-jump connections which depart from $P_{r}$ (since $\left.W^{u}\left(P_{r}\right) \cap \Gamma_{r}=W^{s}\left(Q_{r}\right) \cap \Gamma_{r}\right)$. It is clear that $\gamma_{P_{r} Q_{l}}^{(1)}$ is the only possible candidate (see Figure 7). More precisely, a two-jump combining $\gamma_{P_{l} Q_{r}}^{(1)}$ and $\gamma_{P_{r} Q_{l}}^{(1)}$ is possible if the 'touch-down' point of $\gamma_{P_{l} Q_{r}}^{(1)}$ on $\Gamma_{r}$ has a $z$-coordinate which is larger than the $z$-coordinate of the 'take-off' point of $\gamma_{P_{r} Q_{l}}^{(1)}$. Note that these two orbits are related to each other by the symmetry $\{x \rightarrow-x, y \rightarrow-y\}$. Since the $w$-coordinate of both orbits is (strictly) negative during the jump through the fast field we find by (4.3) that the $z$-coordinate of both orbits decreases monotonically. Thus, the touch-down point is 'below' the take-off point: There cannot be a two-jump orbit if $c_{2}>0$. However, in Theorem 3 we shall show, using a different argument, that there exists a solution connecting $P_{l}$ and $Q_{l}$ with two slow parts near $\Gamma_{l}$ and no slow parts near $\Gamma_{r}$ : It makes a complete circuit through the fast field and does not touch down on $\Gamma_{r}$.

A priori one would assume that the one-jump orbits which jump near the $\{z=0\}$ - 
plane can be used to construct other, new two-jump orbits in the case $c_{2}<0$. By the above arguments it is possible to construct a two-jump orbit which is exponentially close to $\gamma_{P_{l} Q_{r}}^{(1)}$ until it takes off from $\Gamma_{r}$ to follow the one-jump orbit $\gamma_{P_{r} P_{l}}^{(1)}$. However, the thus constructed two-jump homoclinic solution to $P_{l}$ is the symmetric counterpart $\gamma_{P_{l} P_{l}}^{(2,2)}$ of the above-constructed orbit $\gamma_{P_{l} P_{l}}^{(2,1)}$ under the symmetry $\{y \rightarrow-y, t \rightarrow-t, w \rightarrow-w\}$.

We can now formulate the Theorem on $N$-jump orbits:

Theorem 2. Assume that $c_{2}<0$ and $s$ and $c_{1}$ are such that (5.7) holds. Then, for any $N \geq 2$, there are $\mathcal{N}(N)$ distinct $N$-jump heteroclinic or homoclinic orbits $\gamma_{S, T}^{(N, k)}(t)$ between the critical points $S, T \in\left\{P_{l}, P_{r}, Q_{l}, Q_{r}\right\} ; k$ denotes the fact that there can be more than one orbit between $S$ and $T$. The number $\mathcal{N}(N)$ satisfies the recurrence relation

$$
\mathcal{N}(N)=\mathcal{N}(N-1)+\mathcal{N}(N-2) \quad \text { with } \quad \mathcal{N}(1)=8, \quad \mathcal{N}(2)=12 .
$$

These orbits consist of $N+1$ slow passages near $\Gamma_{l, r}$ alternated by $N$ jumps through the fast field and are all exponentially close to the 'skeleton' spanned by the fundamental one-jump solutions constructed in Theorem 1.

Proof. The three-jump orbits are based on the two-jump orbits, just as the two-jump orbits are based on the one-jump orbits. We will start by constructing the orbits $\gamma_{P_{l} Q_{r}}^{(3,1)}(t)$ and $\gamma_{P_{l} P_{r}}^{(3,1)}(t)$; the other three-jump orbits can be found by the symmetries (4.4). We consider, for instance, the heteroclinic orbit $\gamma_{P_{l} Q_{l}}^{(2,1)}(t)$. Let $\mathcal{L}_{2}$ be an exponentially small, one-dimensional neighbourhood of $\gamma_{P_{l} Q_{l}}^{(2,1)}(t) \cap\{x=0\}$ in $\mathcal{F}\left(\mathcal{L}_{1}\right) \cap\{x=0\}$, after the first passage of $\mathcal{F}\left(\mathcal{L}_{1}\right)$ of $\Gamma_{r}$ (where $\mathcal{F}\left(\mathcal{L}_{1}\right)$ is defined above); $\mathcal{L}_{2}$ will play a role similar to $\mathcal{L}_{1}$ in the above construction of the two-jump orbits. We define $\mathcal{F}\left(\mathcal{L}_{2}\right) \subset$ $\mathcal{F}\left(\mathcal{L}_{1}\right)$ as the manifold of orbits through $\mathcal{L}_{2} ; \mathcal{F}\left(\mathcal{L}_{2}\right)$ is separated into two parts by $W^{s}\left(\Gamma_{l}\right)$ with $\gamma_{P_{l} Q_{l}}^{(2,1)}(t)$ as separatrix. Thus, $\mathcal{F}\left(\mathcal{L}_{2}\right)$ is split and twisted by the slow flow near $\Gamma_{l}$ : It becomes a sheet exponentially close to $W^{u}\left(P_{l}\right)$ when it again leaves the neighbourhood of $\Gamma_{l}$. The intersection $\mathcal{F}\left(\mathcal{L}_{2}\right) \cap\{x=0\}$ after the passage of $\Gamma_{l}$ consists of a curve exponentially close to the first intersection of $W^{u}\left(P_{l}\right)$ with $\{x=0\}$ and of the same length as $W^{u}\left(P_{l}\right) \cap\{x=0\}$ (up to $O(\delta)$-terms). Therefore, $\mathcal{F}\left(\mathcal{L}_{2}\right) \cap\{x=0\}$ intersects $W^{s}\left(\Gamma_{r}\right) \cap\{x=0\}$ two times transversally: an intersection exponentially close to $\gamma_{P_{l} Q_{r}}^{(1)}(t) \cap\{x=0\}$ and another exponentially close to $\gamma_{P_{l} P_{r}}^{(1)}(t) \cap\{x=0\}$. By the Hamiltonian character of the flow we know that these intersections must correspond to the three-jump orbits $\gamma_{P_{l} Q_{r}}^{(3,1)}(t)$ and $\gamma_{P_{l} P_{r}}^{(3,1)}(t)$.

It is clear that this construction can be repeated for all $N$ : Based on $\gamma_{P_{l} P_{r}}^{(3,1)}(t)$ we define $\mathcal{L}_{3} \subset \mathcal{F}\left(\mathcal{L}_{2}\right) \cap\{x=0\}$, exponentially close to the third intersection of $\gamma_{P_{l} P_{r}}^{(3,1)}(t)$ with $\{x=0\}$. The manifold $\mathcal{F}\left(\mathcal{L}_{3}\right)$ gets twisted and separated near $\Gamma_{r}$ so that it intersects $W^{s}\left(\Gamma_{l}\right) \cap\{x=0\}$, after its passage of $\Gamma_{r}$, two times: the four-jump orbits $\gamma_{P_{l} P_{l}}^{(4,1)}(t)$ and $\gamma_{P_{l} Q_{l}}^{(4,1)}(t), i=1,2$.

Note that the number $\mathcal{N}(N)$ of $N$-jump orbits increases quite rapidly with $N$. Let's construct $\mathcal{N}(2)$ from $\mathcal{N}(1)=8$ (Theorem 1). If $\gamma^{(1)}$ jumps through the fast field near $\{w=0\}$ then we have shown above that one can construct two two-jump orbits based on this one: one which makes its second jump near $\{z=0\}$ and one which makes its 
second jump again near $\{w=0\}$. If $\gamma^{(1)}$ jumps near $\{z=0\}$, then there only exists one two-jump orbit based on this $\gamma^{(1)}$, which makes its second jump near $\{w=0\}$. Thus, the four one-jump orbits near $\{w=0\}$ lead to eight two-jump orbits; four of them make their last jump near $\{w=0\}$, the other four make their last jump near $\{z=0\}$. The four one-jump orbits near $\{z=0\}$ lead to four two-jump orbits with a second jump near $\{w=0\}$. Thus, as we already found by the symmetries $(4.4), \mathcal{N}(2)=12$, since all these orbits are distinct, by construction.

This method of counting can be used for every transition from $N$ to $N+1$. Define $\mathcal{W}(N)$, resp. $\mathcal{Z}(N)$, the number of $N$-jump orbits of $\mathcal{W}$, resp. $\mathcal{Z}$, type (by definition) which make their final jump through the fast field near $\{w=0\}$, resp. $\{z=0\}$. By the above construction, every $\mathcal{W}$-orbit yields one $\mathcal{W}$-type orbit and one $\mathcal{Z}$-type orbit; a $\mathcal{Z}$-orbit yields a $\mathcal{W}$-orbit; thus,

$$
\left\{\begin{array}{l}
\mathcal{W}(N+1)=\mathcal{W}(N)+\mathcal{Z}(N), \\
\mathcal{Z}(N+1)=\mathcal{W}(N) .
\end{array}\right.
$$

Since $\mathcal{N}(N)=\mathcal{W}(N)+\mathcal{Z}(N)$ we recover (5.9). Note that $\mathcal{N}(N)=4 p_{N+2}$, where $p_{N}$ is the $N$-th Fibonacci number. Thus $\mathcal{N}(N) \approx \frac{1}{2}(1+\sqrt{5}) \mathcal{N}(N-1)$ for large $N$.

Note that the closure of the set of intersections of all $N$-jump orbits with $\{x=0\}$ is a Cantor set of exponentionally small dimension. This can be seen as follows. We take the intersection point $I^{(1)}$ of the orbit $\gamma_{P_{l} P_{r}}^{(1)}(t)$ and $\{x=0\}$ as the base for the construction of a part of this Cantor set; $I^{(1)}$ is the (transversal) intersection of the curves $W^{u}\left(P_{l}\right) \cap\{x=0\}$ and $W^{s}\left(P_{r}\right) \cap\{x=0\}$ in the three-dimensional space $\{x=0\}$ (see Figure 6). By the construction of the orbits $\gamma_{P_{l} P_{l}}^{(2)}(t)$ and $\gamma_{P_{l} Q_{l}}^{(2)}(t)$ (Theorem 2) we know that there exist two points $I_{1,2}^{(2)} \in W^{u}\left(P_{l}\right) \cap\{x=0\}$, exponentially close to $I^{(1)}$. These points are the first intersections of $\gamma_{P_{l} P_{l}}^{(2)}(t)$ and $\gamma_{P_{l} Q_{l}}^{(2)}(t)$ with $\{x=0\}$. Analogously, there are two points $I_{3,4}^{(2)} \in W^{s}\left(P_{r}\right) \cap\{x=0\}$ exponentially close to $I^{(1)}$, corresponding to the second intersections of $\gamma_{P_{r} P_{r}}^{(2)}(t)$ and $\gamma_{Q_{r} P_{r}}^{(2)}(t)$ with $\{x=0\}$. These four new points again are 'surrounded' by intersection points $I_{j}^{(3)}$ of three-jump orbits. The construction of these new points is identical to the construction of the $I_{j}^{(2)}$ points from the point $I^{(1)}$. Note that the ratio of the distance between (for instance) $I_{1}^{(2)}$ and the new points around $I_{1}^{(2)}$, and the distance between $I^{(1)}$ and $I_{1}^{(2)}$ is exponentially small. Thus we can proceed by constructing the points $I_{k}^{(4)}, I_{l}^{(5)}$, etc. Note that at any step one has to 'zoom in' exponentially 'deep' to obtain the next level. The closure of this infinite collection of points $\left\{I_{j}^{(N)}\right\}_{N=1, \ldots, \infty ; j=1, \ldots, j_{N}}$ (where clearly $j_{N} \rightarrow \infty$ as $N \rightarrow \infty$ ) forms a Cantor set of exponentially small (but positive) dimension. Such a set exists near any of the eight base points formed by the intersections of one of the fundamental one-jump orbits (Theorem 1) and $\{x=0\}$. The union of these eight sets again forms a Cantor set.

There are of course more points in this (uncountable) set than the $N$-jump heteroclinic/ homoclinic orbits. One can, for instance, construct many types of different periodic orbits between $\Gamma_{l}$ and $\Gamma_{r}$, which consist of alternating slow and fast parts. Thus, these periodic orbits differ significantly from those found in Section 4.1. None of them can have a counterpart which can be described by the nonlocal system. Note that these periodic 
orbits correspond to limit points of the above-described Cantor set in the $\{x=0\}$ hyperplane. One of the simplest periodic orbits of this type consists of four parts: a slow part exponentially close to $\Gamma_{l}$ and to $\gamma_{P_{l} P_{r}}^{(1)} \cap \gamma_{Q_{r} Q_{l}}^{(1)}$, a fast part near the fast jump of $\gamma_{P_{l} P_{r}}^{(1)}$, a slow part near $\Gamma_{r}$ and $\gamma_{P_{l} P_{r}}^{(1)} \cap \gamma_{Q_{r} Q_{l}}^{(1)}$, and the second fast jump near $\gamma_{Q_{r} Q_{l}}^{(1)}$ (see Figure 9).

So far we have studied orbits which only make jumps directly from $\Gamma_{l}$ to $\Gamma_{r}$, or vice versa. Now we want to construct orbits which make one complete circuit through the fast field. Here we focus, for simplicity, on constructing a heteroclinic orbit from $P_{l}$ to $Q_{l}$ which has two slow parts both near $\Gamma_{l}$ alternated by one 'double' jump which makes a complete circuit through the fast field. This orbit only can be constructed for $c_{1}<0$, where the sign of $c_{2}$ is arbitrary. For $c_{1}>0$ the above orbit generally does not exist. Using the symmetries (4.4) in system (4.3), one can obtain from this orbit other heteroclinic orbits which have two slow parts near $\Gamma_{l}$ or $\Gamma_{r}$ connected by a fast 'double' jump. The idea of the proof of the following theorem is based on the methods developed in [6].

Theorem 3. Assume that $c_{1}<0$ and $s$ and $c_{2}$ are such that (5.7) holds. Then, there exist four heteroclinic orbits $\gamma_{P_{l} Q_{l}}^{(d)}(t), \gamma_{Q_{l} P_{l}}^{(d)}(t), \gamma_{P_{r} Q_{r}}^{(d)}(t)$, and $\gamma_{Q_{r} P_{r}}^{(d)}(t)$. These orbits consist of two slow parts which are both near $\Gamma_{l}\left(\right.$ or $\left.\Gamma_{r}\right)$, alternated by a complete circuit (or a 'double' jump) through the fast field.

Proof. We only construct $\gamma_{P_{l} Q_{l}}^{(d)}(t)$; the other three orbits can be found by applying the symmetries (4.4). Let $\mathcal{L}_{4} \subset W^{u}\left(P_{l}\right) \cap\{x=0\}$ be a (one-dimensional) neighbourhood of $\gamma_{P_{l} Q_{r}}^{(1)} \cap\{x=0\} ; \mathcal{L}_{4}$ intersects $W^{s}\left(Q_{r}\right)$ transversally. Define for $p \in \mathcal{L}_{4}$ the orbit through $p$ by $\gamma_{p}(t) \in W^{u}\left(P_{l}\right)$. Thus for $p_{0}=\mathcal{L}_{4} \cap W^{s}\left(Q_{r}\right), \gamma_{p_{0}}(t)=\gamma_{P_{l} Q_{r}}^{(1)}(t)$. If we take $p_{1} \in \mathcal{L}_{4}$ exponentially close to $p_{0}$, the orbit will follow $\gamma_{p_{0}}$ along $\Gamma_{r}$ for an $O(1)$ distance. Such an orbit $\gamma_{p_{1}}$ will leave the neighbourhood of $\Gamma_{r}$ exponentially close to $W^{u}\left(Q_{r}\right)$ and will still be exponentially close to $W^{u}\left(Q_{r}\right)$ at its next intersection with the hyperplane $\{x=0\}$; we denote this intersection point by $q_{1}$. At this intersection, it will be 'outside' $W^{s}\left(\Gamma_{l}\right)$. Here, an orbit is said to be outside $W^{s}\left(\Gamma_{l}\right)$ when, after the passage near $\Gamma_{l}$, it leaves the neighbourhood of $\Gamma_{l}$ in the direction opposite to $\Gamma_{r}$ (its $x$-coordinate decreases): It cannot return to $\Gamma_{l}$ or $\Gamma_{r}$ and becomes unbounded. On the other hand an orbit is inside $W^{s}\left(\Gamma_{l}\right)$ when it does return to the $\{x=0\}$-hyperplane. In other words, an 'inside' orbit leaves the neighbourhood of $\Gamma_{l}$ near the structure of heteroclinic connections between $\Gamma_{l}$ and $\Gamma_{r}$ which exist in the limit $\delta \downarrow 0$. The fact that an orbit is 'outside' or 'inside' is determined by (5.6) and thus by the sign of $c_{1}$. Now we take $p_{2} \in \mathcal{L}_{4}$ at an $O(1)$ distance from $p_{0}$, where the $z$-coordinate of $p_{2}, p_{2}^{z}$ is larger than the $z$-coordinate of $p_{0}$. Here we also make sure that $p_{2}^{z}$ is not at $O(\delta)$ distance from the $z$-coordinate of $P_{l}$. This assures that the next intersection of $\gamma_{p_{2}}$ with $\{x=0\}$ is inside $W^{s}\left(\Gamma_{l}\right)$; this intersection point is denoted by $q_{2}$. Note that $\gamma_{p_{2}}(t)$ only approaches $\Gamma_{r} O(\sqrt{\delta})$-close. We denote the two-dimensional manifold of orbits $\gamma_{p}$ through $\mathcal{L}_{4}$ by $\mathcal{F}\left(\mathcal{L}_{4}\right)$. From the above it follows that the next intersection of $\mathcal{F}\left(\mathcal{L}_{4}\right)$ with the hyperplane $\{x=0\}$ contains a curve connecting $q_{1}$ and $q_{2}$. Since $q_{1}$ is outside $W^{s}\left(\Gamma_{l}\right)$ and $q_{2}$ is inside $W^{s}\left(\Gamma_{l}\right)$, there exists a $p^{*} \in \mathcal{L}_{4}$ so that the orbit through $p^{*}$ intersects $W^{s}\left(\Gamma_{l}\right)$. Due to the Hamiltonian structure of the flow we know that $p^{*}$ must be on $W^{s}\left(Q_{l}\right)$. Thus we constructed a heteroclinic cycle $\gamma_{p^{*}}=\gamma_{P_{l} Q_{l}}^{(d)}$ with two slow parts near $\Gamma_{l}$ and one fast complete circuit. From the fact that the point $\gamma_{P_{l} P_{r}}^{(1)} \cap\{x=0\}$ is not in the interval 
$\left[p_{1}, p_{2}\right] \subset \mathcal{L}_{4}$, we see that, for $c_{2}<0$, the above orbit is not the one we constructed in Theorem 2, $\gamma_{P_{l} Q_{l}}^{(2)}: \gamma_{P_{l} Q_{l}}^{(d)}$ does not come closer to $\Gamma_{r}$ than $O(\sqrt{\delta})$.

We can show by analogous analysis that such an orbit as constructed above is generally not found for $c_{1}<0$. Again let $p_{0}=\gamma_{P_{l} Q_{r}} \cap\{x=0\}$. Then the orbit through a point on $W^{u}\left(P_{l}\right) \cap\{x=0\}$ which is exponentially close to $p_{0}$ is at its next intersection with the $\{x=0\}$-hyperplane exponentially close to $W^{u}\left(Q_{r}\right)$ and is inside $W^{s}\left(\Gamma_{l}\right)$. However, the orbit through a point which is at $O(1)$ distance from $p_{0}$ (and with a $z$-coordinate which now has to be chosen smaller than $\left.p_{0}^{z}\right)$ is at its next intersection with $\{x=0\}$ also inside $W^{s}\left(\Gamma_{l}\right)$. Thus, the line between these points does, in general, not intersect $W^{s}\left(\Gamma_{l}\right)$ (compare to the 'inside' and 'outside' cases defined in [6]). This implies that such an orbit as constructed in the above theorem generally does not exist for $c_{1}>0$.

Remark 5.1. In all the above theorems we assumed that $s+c_{2}>0$. However, for $s+c_{2}<0$ similar statements hold. Recall that in this case the integrable flow on $\Gamma_{l, r}$ has a 'figure 8' structure: There are two homoclinic orbits to the points $( \pm 1,0,0,0)$ on $\Gamma_{l, r}$ (Figure 4). The points $P_{l, r}, Q_{l, r}$ have become centre points (on $\Gamma_{l, r}$ ). Analogous to Theorem 1 one can prove that there exist eight heteroclinic orbits between the critical points $( \pm 1,0,0,0) \in \Gamma_{l, r}$ which consist of two slow parts near $\Gamma_{l}$ and $\Gamma_{r}$, alternated by one fast jump. There also exist $N$-jump homoclinic orbits, independent of the signs of the coefficients. However, the number of $N$-jumps is not the same as before. There are two two-jump orbits and, for every $N \geq 3$, there exists only one $N$-jump orbit. Thus choosing $s+c_{2}<0$ reduces the number of heteroclinic and homoclinic orbits drastically, although the general behaviour remains the same.

\section{Discussion}

In this paper we derived and studied two different types of modulation equations which describe the same physical phenomena. Pattern formation in a reflection-symmetric system which is subject to two interacting destabilising mechanisms is described by two nonlinearly coupled Ginzburg-Landau equations (1.4). If the natural spatial scales associated to those mechanisms differ significantly (see Figure 1) one can either describe the behaviour near criticality by a singularly perturbed modulation equation (1.5), or one can apply a so-called Landau reduction and derive a nonlocal modulation equation (1.6); see also Metzener and Proctor [22] for the application of this idea. As a necessary consequence of the derivation process we showed that there is an extra, again nonlocal, solvability condition in the nonlocal case (1.7).

Our main goal has been to compare the set of solutions described by the singularly perturbed equation to that of the nonlocal system. We restricted ourselves to the stationary solutions. Note that it is natural to expect stationary patterns in systems with a reflection symmetry, such as convection experiments. For instance, consider the theoretical and analytical study of double-layer convection by Rasenat et al. [25]: Under certain conditions these experiments can be described by the equations studied in this paper (see for instance the neutral curve in Figure 6 in [25]); the experiments performed for this paper exhibit stationary patterns (although the patterns can certainly be nonstationary).

Of course it could be expected that the singularly perturbed equation has a richer set of solutions than the nonlocal reduction. However, in Section 3 we have shown that 
the nonlocal system, combined with the extra condition (1.7), cannot describe any other patterns than purely (spatially) periodic, or quasi-periodic with only two independent frequencies. These patterns are also described by the singularly perturbed system, but this system also governs a very complicated set of 'localised' patterns, corresponding to heteroclinic and homoclinic solutions. These types of patterns are important in the dynamics of the uncoupled Ginzburg-Landau equation (see for instance [28]).

The large families of 'multijump' and 'complete circuit' orbits found in Section 5 only make up a small part of the entire set of possible solutions which have an alternating slowfast structure. The heteroclinic and homoclinic orbits found in Section 5 correspond to only a very small subset of the Cantor sets formed by the intersections $W^{u, s}\left(P_{l, r}, Q_{l, r}\right) \cap$ $\{x=0\}$, which we only briefly discussed in that section. Moreover, we did not pay any attention to connections between the slow manifolds $\Gamma_{l}$ and $\Gamma_{r}$ which are not on the 'energy'-level of the critical points $P_{l, r}, Q_{l, r}$. By the methods developed in this paper it is also possible to show the existence of orbits which connect, through the fast field, corresponding periodic orbits on $\Gamma_{l}$ and $\Gamma_{r}$. Furthermore, the essence of the analysis also works for other values of the $\Omega_{i}$-integrals (see Section 4) than $\Omega_{1}=\Omega_{2}=0$.

This is another aspect of the paper: We have shown that the singularly perturbed Hamiltonian system (1.8) has a very intricately structured phase-space. Moreover, we have been able to unravel much of the structure of this phase-space using in essence topological, or geometrical, methods. These methods are based on the ideas described for instance in [11], [1], and [6].

Thus, the geometrical methods have enabled us to show that the reduction of the singularly perturbed system to the nonlocal system destroys a very large set of 'localised' patterns.

Finally we make just one short remark about the stability of patterns as described by the modulation equations, (1.5) and (1.6). We did not pay any attention to that aspect in this paper. There is much literature on this. We refer to Matkovsky and Volpert [20] where the stability of purely periodic patterns to systems like (1.4), and thus (1.5), has been studied. The same ideas can be used to study corresponding solutions to (1.6). We have not done this in this paper because the analysis is rather straightforward, while the results depend in a complicated manner on the values of the coefficients in the equations. The stability of the quasi-periodic and 'localised' patterns is a much more complicated issue. Only recently has the instability of stationary quasi-periodic patterns to the uncoupled real Ginzburg-Landau equation been proved in [5]. Note that the quasiperiodic solutions found in this paper correspond directly to the quasi-periodic solutions studied in [5]. There are many stability/instability results on 'localised' patterns in an uncoupled Ginzburg-Landau equation. These results only exist for patterns which are much less complicated than most of the ones constructed in this paper. Here, we only refer to [28] and the recent paper [16], in which the approach is also geometrical, and the references given there.

\section{Acknowledgments}

The authors thank Peter Bollerman for his useful remarks on the derivation of the modulation equations in Section 2 and Philip Holmes and Tasso Kaper for comments that improved the presentation of this paper. 


\section{References}

[1] L. Arnold, C. Jones, K. Mischaikow, and G. Raugel (1994) Dynamical Systems, Lecture Notes in Mathematics 1609, Springer-Verlag, New York.

[2] P. Bollerman, A. van Harten, and G. Schneider (1994) On the justification of the GinzburgLandau approximation, in Nonlinear Dynamics and Pattern Formation in the Natural Environment (A. Doelman and A. van Harten, eds.), Pitman Res. Notes in Math. 335, Longman, UK, 20-36.

[3] P. Bollerman (1996) On the Theory of Validity of Amplitude Equations, thesis, Utrecht University, the Netherlands.

[4] A. Doelman (1993) Traveling waves in the complex Ginzburg-Landau equation, J. Nonlin. Sci. 3 225-266.

[5] A. Doelman, R.A. Gardner, and C.K.R.T. Jones (1995) Instability of quasi-periodic solutions of the Ginzburg-Landau equation, Proc. Roy. Soc. Edinburg 125A 501-517.

[6] A. Doelman and P. Holmes (1996) Homoclinic explosions and implosions, Phil. Trans. Roy. Soc. London A 354 845-893.

[7] J. Duan, H.V. Ly, and E.S. Titi (1996) The effects of nonlocal interactions on the dynamics of the Ginzburg-Landau equation, Z. Angew. Math. Phys. 47 433-455.

[8] W. Eckhaus (1983) Relaxation oscillations including a standard chase on French ducks, in Asymptotic Analysis II, Springer Lect. Notes Math. 985 449-494.

[9] W. Eckhaus (1992) On modulation equations of the Ginzburg-Landau type, in ICIAM 91: Proc. 2nd Int. Conf. Ind. Appl. Math. (R.E. O'Malley, ed.), Society for Industrial and Applied Mathematics, Philadelphia, 83-98.

[10] W. Eckhaus (1993) The Ginzburg-Landau manifold is an attractor, J. Nonlin. Sci. 3 329-348.

[11] N. Fenichel (1979) Geometric singular perturbation theory for ordinary differential equations, J. Diff. Eq. 31 53-98.

[12] A. van Harten (1991) On the validity of Ginzburg-Landau's equation, J. Nonlin. Sci. $1397-$ 422.

[13] D.R. Jenkins (1985) Non-linear interaction of morphological and convective instabilities during solidification of a binary alloy, I.M.A. J. Appl. Math. 35 145-157.

[14] C.K.R.T. Jones and N. Kopell (1994) Tracking invariant manifolds with differential forms in singularly perturbed systems, J. Diff. Eq. 108 64-88.

[15] C.K.R.T. Jones, T. Kaper, and N. Kopell (1996) Tracking invariant manifolds up to exponentially small errors, SIAM J. Math. An. 27 558-577.

[16] T. Kapitula (1996) Existence and stability of singular heteroclinic orbits for the GinzburgLandau equation, Nonlinearity $9669-685$.

[17] E. Knobloch and J. De Luca (1990) Amplitude equations for travelling wave convection, Nonlinearity 3 975-980.

[18] G. Manogg and P. Metzener (1994) Interaction of modes with disparate scales in RayleighBénard convection, in Nonlinear Dynamics and Pattern Formation in the Natural Environment (A. Doelman and A. van Harten, eds.), Pitman Res. Notes in Math. 335, Longman, Harlow, Essex, UK, 188-205.

[19] B.J. Matkovsky and V. Volpert (1992) Coupled nonlocal complex Ginzburg-Landau equations in gasless combustion, Physica 54D 203-219.

[20] B.J. Matkovsky and V. Volpert (1993) Stability of plane wave solutions of complex GinzburgLandau equations, Quart. Appl. Math. 51 265-281.

[21] G.J. Merchant and S.H. Davis (1990) Morphological instability in rapid directional solidification, Acta Metall. Mater. 38 2683-2693.

[22] P. Metzener and M.R.E. Proctor (1992) Interaction of patterns with disparate scales, Eur. J. Mech. B/Fluids 11 759-778.

[23] R.D. Pierce and C.E. Wayne (1995) On the validity of mean-field amplitude equations for counterpropagating wavetrains, Nonlinearity 8 769-779. 
[24] M.R.E. Proctor and C.A. Jones (1988) The interaction of two spatially resonant patterns in thermal convection, Part 1. Exact 2:1 resonance, J. Fluid Mech. 188 301-335.

[25] S. Rasenat, F. Busse, and I. Rehberg (1989) A theoretical and experimental study of doublelayer convection, J. Fluid Mech. 199 519-540.

[26] D.S. Riley and S.H. Davis (1990) Long-wave interaction in morphological and convective instabilities, I.M.A. J. Appl. Math. 45 267-285.

[27] C. Robinson (1983) Sustained resonance for a nonlinear system with slowly varying coefficients, SIAM Math. An. 14 847-860.

[28] W. van Saarloos and P. C. Hohenberg (1992) Fronts, pulses, sources and sinks in generalized complex Ginzburg-Landau equations, Physica 56D 303-367.

[29] R.M.J. Schielen and A. Doelman (1996) Modulation equations for spatially periodic systems: Derivation and solutions, preprint.

[30] J.T. Stuart (1958) On the non-linear mechanics of hydrodynamic stability, J. Fluid Mech. 4 $1-21$.

[31] G. Vittori and P. Blondeaux (1992) Sand ripples under sea waves, Part 3. Brick pattern ripple formation, J. Fluid Mech. 239 23-45.

[32] S. Wiggins (1988) Global Bifurcations and Chaos, Springer-Verlag, New York. 